\title{
A Possible Radiation-Induced Transition from Monazite-(Ce) to Xenotime-(Y)
}

\author{
M. Mashrur Zaman and Sytle M. Antao* ${ }^{\mathbb{D}}$
}

\section{check for}

updates

Citation: Zaman, M.M.; Antao, S.M A Possible Radiation-Induced Transition from Monazite-(Ce) to Xenotime-(Y). Minerals 2021, 11, 16. https: / / dx.doi.org/10.3390/ $\min 11$ 010016

Received: 10 December 2020 Accepted: 23 December 2020 Published: 25 December 2020

Publisher's Note: MDPI stays neutral with regard to jurisdictional claims in published maps and institutional affiliations.

Copyright: () 2020 by the authors. Licensee MDPI, Basel, Switzerland. This article is an open access article distributed under the terms and conditions of the Creative Commons Attribution (CC BY) license (https: / / creativecommons.org/ licenses/by/4.0/).
Department of Geoscience, University of Calgary, Calgary, AB T2N 1N4, Canada; mmzaman@ucalgary.ca

* Correspondence: antao@ucalgary.ca

\begin{abstract}
This study examines two pegmatitic monazite samples ( $2 \mathrm{a}$ and $4 \mathrm{~b}$, these numbers are related to a previous study) to determine their crystal chemistry and effects of internal radiation damage using synchrotron high-resolution powder X-ray diffraction and electron-probe micro-analysis. Both the huttonite and cheralite substitutions are discussed. Rietveld structure refinement of sample $2 \mathrm{a}$ shows three different phases [2a = monazite-(Ce), $2 \mathrm{~b}=$ monazite-(Ce), and $2 \mathrm{c}=$ xenotime- $(\mathrm{Y})$ ] with distinct structural parameters. The changes among the unit-cell parameters between the two monazite-(Ce) phases is more pronounced in the $a$ followed by the $b$ and $c$ unit-cell parameters. Sample $4 \mathrm{a}$ is a single-phase monazite-(Sm) that contains 0.164 apfu Th. Phase $2 \mathrm{c}$ with space group $I 4_{1}$ /amd arises from redistribution of $\mathrm{La}, \mathrm{Ce}, \mathrm{Pr}, \mathrm{Nd}, \mathrm{Sm}, \mathrm{Gd}, \mathrm{Dy}, \mathrm{Si}$, and $\mathrm{Y}$ atoms from those in monazite (space group $P 2_{1} / n$ ). A possible cause for the phase transition from monazite-(Ce) to xenotime-( $(\mathrm{Y})$ is $\alpha$-radiation events over a long geological time. However, other chemical processes cannot be ruled out as a cause for the transition.
\end{abstract}

Keywords: monazite; xenotime; radiation damage; Rietveld refinements; crystal structure; structural variations

\section{Introduction}

Both monazite and xenotime are phosphate minerals that contain rare-earth elements (REE). They have the general formula $A \mathrm{PO}_{4}$, where $A=\mathrm{REE}$. Monazite is monoclinic with space group $P 2_{1} / n$ and is isostructural to huttonite, the high $\mathrm{P}$-high T polymorph of $\mathrm{ThSiO}_{4}$ [1-3]. Xenotime is tetragonal with space group $I 4_{1} /$ amd, and is isostructural with thorite $\left(\mathrm{ThSiO}_{4}\right)$, which is the low $\mathrm{P}$-low $\mathrm{T}$ polymorph of $\mathrm{ThSiO}_{4}$. Thorite is also isostructural with zircon $\left(\mathrm{ZrSiO}_{4}\right)$. Structural trends in zircon and monazite samples from various localities were recently discussed $[4,5]$.

Monazite contains light rare earth elements (LREE) and has a general formula (Ce, $\mathrm{La}, \mathrm{Nd}, \mathrm{Sm}, \mathrm{Y}, \mathrm{Th}) \mathrm{PO}_{4}$. The lanthanide series $\left(\mathrm{Ln}^{3+}\right)$ is subdivided into LREE-La to Nd, middle rare earth elements (MREE) — Sm to Dy, and heavy rare earth elements (HREE) - Ho to Lu. LREE are common in the Earth's crust compared to HREE. The ionic radii of $\mathrm{Ln}^{3+}$ cations decrease as the atomic number increases. This change in $\mathrm{Ln}^{3+}$ ionic radii control the crystal structure of REE $\left(\mathrm{PO}_{4}\right)$. REE in monazite samples from different sources (e.g., pegmatites, granites, etc.) contain $\mathrm{La}, \mathrm{Nd}, \mathrm{Pr}, \mathrm{Ce}, \mathrm{Sm}, \mathrm{Gd}$, and Y [6]. Most metamorphic monazite samples have a composition close to $\left(\mathrm{Ce}_{0.43} \mathrm{La}_{0.20} \mathrm{Nd}_{0.17}\right) \mathrm{PO}_{4}$ [7]. Although monazite contains $\mathrm{Ce}$ atoms, this is not always the dominant cation. Based on the dominant cation, samples are called monazite-(Ce), monazite-(Sm), monazite-(Nd), monazite-(La), etc. Monazite is an accessory mineral in intermediate to high grade metamorphic rocks, biotite granites, syenitic and granitic pegmatites, quartz veins, and carbonatites [8,9]. It also occurs as a detrital mineral in placer deposits, beach sands, and river sands.

Both monazite and xenotime contain $\mathrm{PO}_{4}$ tetrahedral groups. Monazite contains $\mathrm{AO}_{9}$ polyhedra, whereas xenotime contains $\mathrm{AO}_{8}$ polyhedra $[5,10,11]$. Both structures contain alternating polyhedra and tetrahedra that form chains sharing O-O edges parallel to [001]. The xenotime structure has a higher symmetry than the monazite structure (Figure 1). 


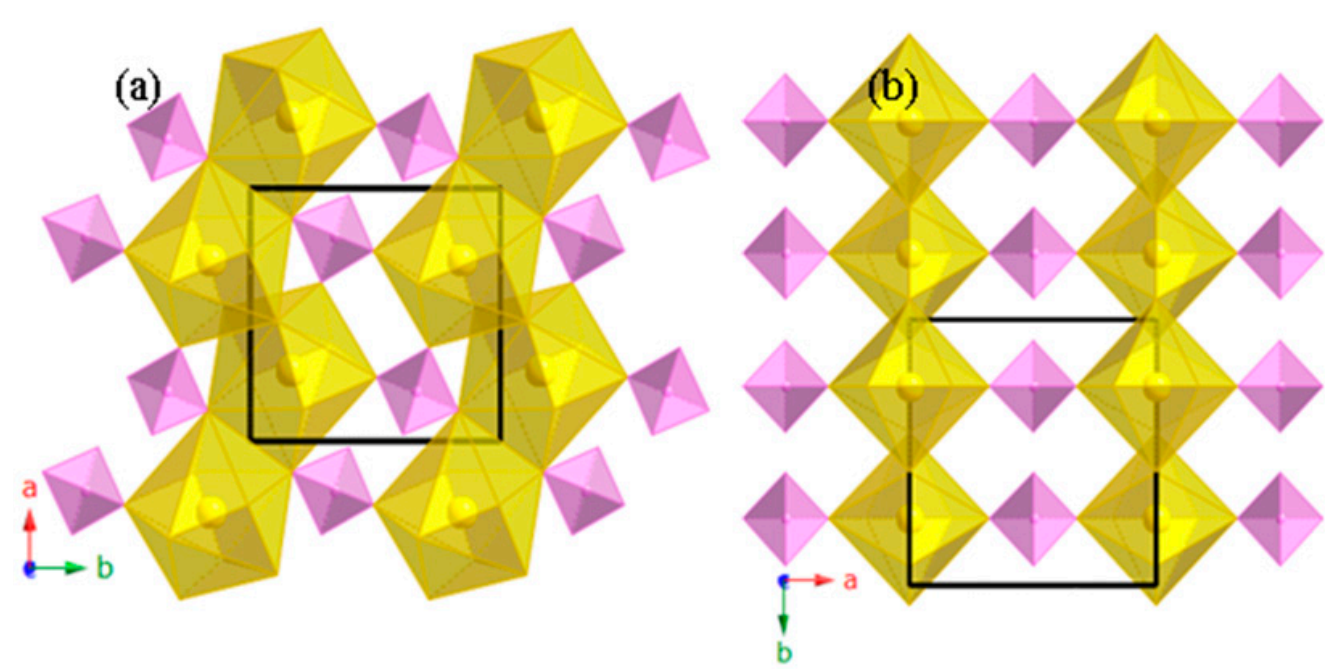

Figure 1. The structures of (a) monazite (space group $P 2_{1} / n$ ) and (b) xenotime (space group $I 4_{1} / a m d$ ) projected down [001] with unit cells outlined in black. The two structures are similar and the transition from monazite to xenotime results in a more symmetrical structure. Rotation of the $\mathrm{PO}_{4}$ tetrahedra (purple) in (a) gives rise to more symmetrical features in (b). Polyhedra $\mathrm{AO}_{9}$ in (a) and $\mathrm{AO}_{8}$ in (b) are shown in yellow.

Both monazite and xenotime experience internal radiation doses because they contain small amounts of thorium (Th) and uranium (U) atoms, but they do not carry any effects of radiation damage [12-15], but radiation damage in monazite was reported [16]. Monazite has the ability to heal its crystal structure between $373-473 \mathrm{~K}[15,17]$. Another potential reason could be its structural differences from that of zircon. Monazite has P-O distances that are shorter and stronger than the $\mathrm{Si}-\mathrm{O}$ distances in zircon that may promote the resistance to radiation damage [18]. Two different monazite phases were found in a crystal [15]: phase e1 is well crystalline with trapped helium atoms that cause an increase in unit-cell parameters, whereas phase e 2 represents a distorted lattice, which is referred to as "old alpha recoil tracks" that is generated by the recoil atoms after a radioactive decay event. Two different monazite phases $\mathrm{f} 1$ and $\mathrm{f} 2$ were found in another study [19]. Except for the unit-cell parameters, in neither of these two previous studies $[12,15]$ were the crystal structures refined. The degree of radiation damage in minerals depends mainly on the ratio of damage accumulation and thermal annealing rates. If the recovery processes dominate, the crystallinity is preserved, even at a low temperature [20].

This study investigates the crystal-chemical properties and effects of radiation doses in two monazite samples using synchrotron high-resolution powder X-ray diffraction (HRPXRD) and electron-probe microanalysis (EPMA). Our sample 2a contains three phases: phases $2 a$ and $2 b$ are monazite- $(\mathrm{Ce})$ and phase $2 c$ is xenotime- $(\mathrm{Y})$. The presence of xenotime(Y) may indicate a radiation-induced transition from monazite to xenotime. However, other chemical processes cannot be ruled out as a cause for the transition. Our sample $4 \mathrm{a}$ is a single monazite-( $\mathrm{Sm})$ phase. Both samples $2 \mathrm{a}$ and $4 \mathrm{a}$ were examined previously using single-crystal X-ray diffraction (SCXRD) and they were called samples 2 and 4 , respectively $[5,21]$.

\section{Experimental Methods}

\subsection{Sample Description}

Two pegmatitic monazite samples 2a (Ce-dominated) and 4a (Sm-dominated) were used in this study and their description and occurrence are summarized in Table 1. Fragments of monazite were separated from the two samples with a knife. The crystal fragments were examined with a stereomicroscope and high purity, optically clear, and inclusion-free fragments were picked for EPMA and synchrotron HRPXRD studies. Because sample 2a 
contains several phases, HRPXRD is a more suitable technique than SCXRD to get more detailed structural information including a micro-strain.

Table 1. Monazite sample information.

\begin{tabular}{ccc}
\hline Sample No. & Locality & Description and Occurrence \\
\hline $2 \mathrm{a}$ & Iveland, Norway & $\begin{array}{c}\text { Massive brown monazite-(C) occurs in a } \\
\text { quartz pegmatitic rock. }\end{array}$ \\
\hline 4a & Gunnison County, Colorado, USA & $\begin{array}{c}\text { Massive brown monazite-(Sm) occurs } \\
\text { with cleavelandite feldspar and lepidolite } \\
\text { from the brown Derby-1 pegmatite. }\end{array}$ \\
\hline
\end{tabular}

\subsection{Electron-Probe Microanalysis (EPMA)}

The chemical composition of the monazite samples was obtained using a JEOL JXA8200WD-ED electron-probe microanalyzer (Akishima, Tokyo, Japan). The JEOL operating program on a Solaris platform was used for ZAF (atomic number, absorption, and fluorescence) correction and data reduction. The wavelength-dispersive (WD) analysis was conducted quantitatively using an accelerated voltage of $15 \mathrm{kV}$, a beam current of $2 \times 10^{-8} \mathrm{~A}$, and a beam diameter of $5 \mu \mathrm{m}$. Peak overlapping problems in the elemental analysis of monazite are very common and were solved following the method previously described [22]. Various minerals and compounds were used as standards $\left(\mathrm{CePO}_{4}\right.$ for $\mathrm{Ce}$ and $\mathrm{P}, \mathrm{NdPO}_{4}$ for $\mathrm{Nd}, \mathrm{YPO}_{4}$ for $\mathrm{Y}, \mathrm{ThO}_{2}$ for Th, $\mathrm{LaPO}_{4}$ for $\mathrm{La}, \mathrm{SmPO}_{4}$ for Sm, $\mathrm{PrPO}_{4}$ for $\mathrm{Pr}, \mathrm{GdPO}_{4}$ for $\mathrm{Gd}, \mathrm{DyPO}_{4}$ for $\mathrm{Dy}, \mathrm{EuPO}_{4}$ for $\mathrm{Eu}, \mathrm{TbPO}_{4}$ for $\mathrm{Tb}$, zircon for $\mathrm{Si}, \mathrm{Cr}$-augite for $\mathrm{Ca}$, barite for $\mathrm{S}$, pyromorphite for $\mathrm{Pb}, \mathrm{UO}_{2}$ for $\mathrm{U}$, and hornblende for $\mathrm{Fe}$ ). Seventeen spots (S1-S17) were analyzed for each sample. The oxide wt. \% and the calculated atom per formula unit (apfu) based on four oxygen $(\mathrm{O})$ atoms are given in Tables 2 and 3. A summary of the chemical composition for samples $2 \mathrm{a}$ and $4 \mathrm{a}$ is also given (Table 4). Three energy dispersive spectra (EDS) were also obtained with EPMA.

\subsection{Age Determination and Radiation Doses Calculation}

The ages of both monazite samples were unknown. Separate data for the concentrations of $\mathrm{U}, \mathrm{Th}$, and $\mathrm{Pb}$ from nine spots (A1-A9) for samples $2 \mathrm{a}$ and 4 a were collected using the same experimental conditions as used for the full data collection. The chemical age (T) of the samples were determined using the following relation [23].

$\mathrm{Pb}=(\mathrm{Th} / 232)\left[\exp \left(\lambda^{232} \times T\right)-1\right] \times 208+(\mathrm{U} / 238.04) \times 0.9928\left[\exp \left(\lambda^{238} \times T\right)-1\right] \times 206+(\mathrm{U} / 238.04) \times 0.0072\left[\exp \left(\lambda^{235} \times T\right)-1\right] \times 207$

where $\mathrm{Pb}, \mathrm{U}$, and $\mathrm{Th}=$ the concentrations in $\mathrm{ppm}$, and $\lambda^{235}, \lambda^{238}$, and $\lambda^{232}=$ the radioactive decay constants (year ${ }^{-1}$ ) of ${ }^{235} \mathrm{U},{ }^{238} \mathrm{U}$, and ${ }^{232} \mathrm{Th}$, respectively.

Assumptions used in the calculations are that the initial concentration of $\mathrm{Pb}$ must be negligible, so all $\mathrm{Pb}$ are radiogenic and the concentrations of $\mathrm{U}$ and Th must not be modified by other means except radioactive decay.

The $\alpha$-radiation doses were calculated using Equation (2) from Murakami et al. [24], which is modified from Holland and Gottfried [25]:

$$
D=8 N_{1}\left[\exp \left(\lambda^{238} \times T\right)-1\right]+7 N_{2}\left[\exp \left(\lambda^{235} \times T\right)-1\right]+6 N_{3}\left[\exp \left(\lambda^{232} \times T\right)-1\right]
$$

where $T$ = is the age of the sample, $D=$ the dose in $\alpha$-decay events $/ \mathrm{mg}, N_{1}, N_{2}$, and $N_{3}=$ the present numbers of ${ }^{238} \mathrm{U},{ }^{235} \mathrm{U}$, and ${ }^{232} \mathrm{Th}$ in atoms $/ \mathrm{mg}$, and $\lambda^{235}, \lambda^{238}$, and $\lambda^{232}=$ the radioactive decay constants (year ${ }^{-1}$ ) of ${ }^{235} \mathrm{U},{ }^{238} \mathrm{U}$, and ${ }^{232} \mathrm{Th}$, respectively. Concentrations of $\mathrm{U}, \mathrm{Th}$, and $\mathrm{Pb}(\mathrm{ppm})$ from nine EPMA spots, calculated age, and $\alpha$-radiation doses for samples $2 \mathrm{a}$ and $4 \mathrm{a}$ are given in Table 5. 
Table 2. Monazite-(Ce): EPMA data from 17 spots (S1 to S17) and their average (Av) for sample 2a.

\begin{tabular}{|c|c|c|c|c|c|c|c|c|c|c|c|c|c|c|c|c|c|c|}
\hline Oxides & S1 & S2 & S3 & S4 & S5 & S6 & S7 & S8 & S9 & S10 & S11 & S12 & S13 & S14 & S15 & S16 & S17 & Av \\
\hline $\mathrm{La}_{2} \mathrm{O}_{3}$ & 7.75 & 7.74 & 7.55 & 7.74 & 7.68 & 7.65 & 7.91 & 7.65 & 7.90 & 7.63 & 8.05 & 9.76 & 9.95 & 9.39 & 8.39 & 10.20 & 8.90 & 8.34 \\
\hline $\mathrm{Ce}_{2} \mathrm{O}_{3}$ & 22.80 & 22.68 & 21.84 & 22.61 & 22.28 & 22.04 & 22.62 & 22.42 & 22.78 & 22.33 & 23.66 & 28.26 & 27.95 & 27.15 & 24.22 & 28.40 & 26.36 & 24.14 \\
\hline $\mathrm{Pr}_{2} \mathrm{O}_{3}$ & 3.16 & 3.15 & 3.32 & 3.10 & 3.14 & 3.29 & 2.97 & 3.25 & 3.26 & 3.35 & 3.30 & 3.81 & 4.06 & 3.86 & 3.30 & 3.68 & 4.13 & 3.42 \\
\hline $\mathrm{Nd}_{2} \mathrm{O}_{3}$ & 15.03 & 14.97 & 15.27 & 15.07 & 14.83 & 15.35 & 14.92 & 15.31 & 15.16 & 15.20 & 15.15 & 17.70 & 16.99 & 16.92 & 15.57 & 17.35 & 17.30 & 15.77 \\
\hline $\mathrm{Sm}_{2} \mathrm{O}_{3}$ & 3.96 & 4.10 & 4.12 & 4.24 & 4.08 & 4.01 & 3.88 & 4.01 & 3.96 & 4.10 & 4.00 & 4.54 & 4.47 & 4.24 & 4.31 & 4.42 & 4.66 & 4.18 \\
\hline $\mathrm{Eu}_{2} \mathrm{O}_{3}$ & bdl & bdl & bdl & bdl & bdl & bdl & bdl & bdl & bdl & bdl & bdl & bdl & bdl & bdl & bdl & bdl & bdl & \\
\hline $\mathrm{Gd}_{2} \mathrm{O}_{3}$ & 2.44 & 2.50 & 2.06 & 2.25 & 2.05 & 2.16 & 2.20 & 2.33 & 2.10 & 2.30 & 2.34 & 1.98 & 1.91 & 1.80 & 1.98 & 1.96 & 2.17 & 2.15 \\
\hline $\mathrm{Tb}_{2} \mathrm{O}_{3}$ & 0.05 & 0.02 & 0.08 & 0.03 & 0.05 & 0.08 & 0.14 & 0.11 & 0.13 & 0.06 & 0.04 & bdl & bdl & bdl & 0.029 & bdl & bdl & 0.07 \\
\hline $\mathrm{Dy}_{2} \mathrm{O}_{3}$ & 0.74 & 0.66 & 0.80 & 0.61 & 0.78 & 0.79 & 0.74 & 0.79 & 0.75 & 0.85 & 0.79 & 0.24 & 0.25 & 0.21 & 0.66 & 0.16 & 0.32 & 0.60 \\
\hline $\mathrm{Y}_{2} \mathrm{O}_{3}$ & 3.82 & 4.16 & 4.07 & 3.98 & 3.78 & 3.79 & 3.85 & 3.92 & 3.93 & 4.15 & 3.90 & 0.38 & 0.60 & 0.70 & 3.10 & 0.28 & 0.92 & 2.90 \\
\hline $\mathrm{FeO}$ & bdl & $\mathrm{bdl}$ & bdl & bdl & $\mathrm{bdl}$ & bdl & bdl & $\mathrm{bdl}$ & $\mathrm{bdl}$ & bdl & 0.028 & 0.00 & 0.01 & 0.14 & bdl & 0.04 & 0.20 & 0.07 \\
\hline $\mathrm{P}_{2} \mathrm{O}_{5}$ & 26.44 & 26.89 & 26.84 & 26.73 & 26.01 & 26.48 & 26.03 & 27.14 & 26.51 & 26.78 & 27.31 & 29.52 & 28.93 & 27.56 & 26.80 & 29.39 & 28.52 & 27.29 \\
\hline $\mathrm{SiO}_{2}$ & 2.02 & 1.94 & 1.96 & 2.12 & 2.05 & 2.03 & 2.02 & 2.06 & 2.08 & 1.98 & 1.74 & 0.19 & 0.43 & 0.47 & 1.29 & 0.21 & 0.74 & 1.49 \\
\hline $\mathrm{SO}_{3}$ & bdl & 0.10 & 0.03 & 0.01 & bdl & 0.02 & 0.00 & 0.09 & 0.07 & bdl & bdl & bdl & bdl & 0.04 & 0.03 & bdl & 0.14 & 0.05 \\
\hline $\mathrm{ThO}_{2}$ & 8.97 & 8.08 & 8.80 & 8.47 & 8.82 & 8.65 & 8.86 & 8.71 & 9.02 & 8.23 & 7.55 & 2.12 & 3.34 & 4.22 & 6.64 & 2.01 & 3.34 & 6.81 \\
\hline $\mathrm{UO}_{2}$ & 0.27 & 0.36 & 0.49 & 0.31 & 0.38 & 0.36 & 0.35 & 0.26 & 0.29 & 0.40 & 0.46 & 0.11 & 0.28 & 0.60 & 0.17 & 0.12 & 0.15 & 0.32 \\
\hline $\mathrm{PbO}$ & 0.25 & 0.30 & 0.30 & 0.28 & 0.30 & 0.29 & 0.29 & 0.29 & 0.30 & 0.30 & 0.16 & 0.02 & 0.15 & 0.15 & 0.16 & 0.03 & 0.15 & 0.22 \\
\hline $\begin{array}{l}\text { Total } \\
\text { apfu * }\end{array}$ & 98.01 & 97.87 & 97.76 & 97.81 & 96.44 & 97.25 & 97.01 & 98.61 & 98.43 & 97.92 & 98.70 & 98.87 & 99.64 & 97.72 & 96.97 & 98.48 & 98.28 & 97.99 \\
\hline $\mathrm{La}$ & 0.117 & 0.116 & 0.113 & 0.116 & 0.118 & 0.116 & 0.121 & 0.113 & 0.118 & 0.114 & 0.119 & 0.143 & 0.146 & 0.143 & 0.128 & 0.151 & 0.132 & 0.125 \\
\hline $\mathrm{Pr}$ & 0.047 & 0.047 & 0.049 & 0.046 & 0.047 & 0.049 & 0.045 & 0.048 & 0.048 & 0.050 & 0.048 & 0.055 & 0.059 & 0.058 & 0.050 & 0.054 & 0.060 & 0.051 \\
\hline $\mathrm{Nd}$ & 0.219 & 0.217 & 0.222 & 0.219 & 0.220 & 0.225 & 0.221 & 0.220 & 0.220 & 0.221 & 0.218 & 0.252 & 0.242 & 0.249 & 0.229 & 0.248 & 0.248 & 0.229 \\
\hline $\mathrm{Sm}$ & 0.056 & 0.057 & 0.058 & 0.059 & 0.058 & 0.057 & 0.055 & 0.056 & 0.055 & 0.057 & 0.055 & 0.062 & 0.062 & 0.060 & 0.061 & 0.061 & 0.064 & 0.059 \\
\hline $\mathrm{Gd}$ & 0.033 & 0.034 & 0.028 & 0.030 & 0.028 & 0.029 & 0.030 & 0.031 & 0.028 & 0.031 & 0.031 & 0.026 & 0.025 & 0.025 & 0.027 & 0.026 & 0.029 & 0.029 \\
\hline $\mathrm{Tb}$ & 0.001 & - & 0.001 & - & 0.001 & 0.001 & 0.002 & 0.001 & 0.002 & 0.001 & - & - & - & - & - & - & - & 0.001 \\
\hline Dy & 0.010 & 0.009 & 0.011 & 0.008 & 0.010 & 0.010 & 0.010 & 0.010 & 0.010 & 0.011 & 0.010 & 0.003 & 0.003 & 0.003 & 0.009 & 0.002 & 0.004 & 0.008 \\
\hline Y & 0.083 & 0.090 & 0.088 & 0.086 & 0.084 & 0.083 & 0.085 & 0.084 & 0.085 & 0.090 & 0.084 & 0.008 & 0.013 & 0.015 & 0.068 & 0.006 & 0.020 & 0.063 \\
\hline $\mathrm{Ca}$ & 0.013 & 0.009 & 0.010 & 0.011 & 0.010 & 0.011 & 0.010 & 0.012 & 0.010 & 0.011 & 0.010 & 0.010 & 0.014 & 0.012 & 0.014 & 0.009 & 0.012 & 0.011 \\
\hline $\mathrm{Fe}$ & - & - & - & - & - & - & - & - & - & - & 0.001 & - & - & 0.005 & - & 0.001 & 0.007 & 0.001 \\
\hline $\mathrm{P}$ & 0.915 & 0.923 & 0.924 & 0.920 & 0.914 & 0.919 & 0.912 & 0.923 & 0.912 & 0.922 & 0.931 & 0.996 & 0.978 & 0.962 & 0.936 & 0.995 & 0.970 & 0.938 \\
\hline $\mathrm{Si}$ & 0.082 & 0.079 & 0.080 & 0.086 & 0.085 & 0.083 & 0.084 & 0.083 & 0.084 & 0.080 & 0.070 & 0.008 & 0.017 & 0.020 & 0.053 & 0.008 & 0.030 & 0.061 \\
\hline $\mathrm{S}$ & - & 0.003 & 0.001 & - & - & 0.001 & - & 0.003 & 0.002 & - & - & - & - & 0.001 & 0.001 & - & 0.004 & 0.001 \\
\hline Th & 0.083 & 0.075 & 0.081 & 0.078 & 0.083 & 0.081 & 0.083 & 0.080 & 0.083 & 0.076 & 0.069 & 0.019 & 0.030 & 0.040 & 0.062 & 0.018 & 0.031 & 0.063 \\
\hline $\mathrm{U}$ & 0.002 & 0.003 & 0.004 & 0.003 & 0.003 & 0.003 & 0.003 & 0.002 & 0.003 & 0.004 & 0.004 & 0.001 & 0.002 & 0.006 & 0.002 & 0.001 & 0.001 & 0.003 \\
\hline $\mathrm{Pb}$ & 0.003 & 0.003 & 0.003 & 0.003 & 0.003 & 0.003 & 0.003 & 0.003 & 0.003 & 0.003 & 0.002 & - & 0.002 & 0.002 & 0.002 & - & 0.002 & 0.002 \\
\hline Total & 2.006 & 2.000 & 1.999 & 2.002 & 2.004 & 2.002 & 2.006 & 1.998 & 2.004 & 2.004 & 2.002 & 1.997 & 2.003 & 2.009 & 2.008 & 1.997 & 2.002 & 2.003 \\
\hline
\end{tabular}

$*$ apfu $=$ atom per formula unit based on $4 \mathrm{O}$ atoms. bdl $=$ below detection limits. 
Table 3. Monazite-(Sm): EPMA data from 17 spots (S1 to S17) and their average (Av) for sample 4a.

\begin{tabular}{|c|c|c|c|c|c|c|c|c|c|c|c|c|c|c|c|c|c|c|}
\hline Oxides & S1 & S2 & S3 & S4 & S5 & S6 & S7 & S8 & S9 & S10 & S11 & S12 & S13 & S14 & S15 & S16 & S17 & Av \\
\hline $\mathrm{La}_{2} \mathrm{O}_{3}$ & 3.91 & 3.98 & 4.04 & 3.76 & 3.97 & 4.08 & 3.83 & 3.73 & 3.81 & 3.81 & 3.80 & 3.89 & 3.88 & 3.85 & 3.98 & 4.23 & 4.03 & 3.92 \\
\hline $\mathrm{Ce}_{2} \mathrm{O}_{3}$ & 12.12 & 12.24 & 12.55 & 12.03 & 12.71 & 12.71 & 12.24 & 12.06 & 12.31 & 12.43 & 12.42 & 12.57 & 12.69 & 12.76 & 12.23 & 12.84 & 12.52 & 12.44 \\
\hline $\mathrm{Pr}_{2} \mathrm{O}_{3}$ & 1.63 & 1.67 & 1.88 & 1.72 & 1.96 & 1.65 & 1.82 & 1.86 & 1.78 & 1.74 & 1.92 & 1.82 & 1.87 & 1.92 & 1.87 & 1.89 & 1.85 & 1.81 \\
\hline $\mathrm{Nd}_{2} \mathrm{O}_{3}$ & 7.11 & 6.99 & 7.31 & 7.32 & 7.16 & 7.05 & 7.36 & 7.04 & 7.07 & 7.05 & 7.12 & 7.17 & 7.16 & 7.27 & 7.09 & 7.20 & 7.08 & 7.15 \\
\hline $\mathrm{Eu}_{2} \mathrm{O}_{3}$ & bdl & bdl & bdl & bdl & bdl & bdl & bdl & $\mathrm{bdl}$ & bdl & bdl & bdl & $\mathrm{bdl}$ & bdl & bdl & bdl & bdl & bdl & 0.00 \\
\hline $\mathrm{Gd}_{2} \mathrm{O}_{3}$ & 5.73 & 5.56 & 5.47 & 5.74 & 5.45 & 5.68 & 5.76 & 5.47 & 5.80 & 5.40 & 5.77 & 5.66 & 5.05 & 5.60 & 5.14 & 5.28 & 5.60 & 5.54 \\
\hline $\mathrm{Tb}_{2} \mathrm{O}_{3}$ & bdl & bdl & bdl & bdl & bdl & bdl & bdl & bdl & bdl & bdl & bdl & 0.06 & 0.07 & 0.02 & 0.10 & bdl & 0.09 & 0.02 \\
\hline $\mathrm{Dy}_{2} \mathrm{O}_{3}$ & 0.34 & 0.27 & 0.28 & 0.29 & 0.25 & 0.27 & 0.27 & 0.38 & 0.39 & 0.34 & 0.27 & 0.39 & 0.37 & 0.35 & 0.31 & 0.20 & 0.24 & 0.31 \\
\hline $\mathrm{Y}_{2} \mathrm{O}_{3}$ & 0.94 & 0.78 & 0.72 & 0.73 & 0.40 & 0.50 & 0.59 & 0.39 & 0.41 & 0.67 & 0.73 & 0.41 & 0.66 & 0.78 & 0.88 & 0.61 & 0.70 & 0.64 \\
\hline $\mathrm{FeO}$ & bdl & bdl & bdl & bdl & bdl & bdl & bdl & 0.03 & bdl & bdl & bdl & bdl & bdl & bdl & bdl & bdl & 0.01 & 0.00 \\
\hline $\mathrm{P}_{2} \mathrm{O}_{5}$ & 28.04 & 27.85 & 27.99 & 28.19 & 28.28 & 28.15 & 27.78 & 27.08 & 28.90 & 27.71 & 27.94 & 28.02 & 27.80 & 27.92 & 28.16 & 27.64 & 27.87 & 27.96 \\
\hline $\mathrm{SiO}_{2}$ & 1.24 & 1.26 & 1.28 & 1.25 & 1.15 & 1.20 & 1.32 & 1.19 & 1.01 & 1.26 & 1.22 & 1.25 & 1.18 & 1.21 & 1.14 & 1.15 & 1.24 & 1.21 \\
\hline $\mathrm{SO}_{3}$ & 0.02 & bdl & bdl & bdl & bdl & bdl & 0.18 & bdl & 0.03 & 0.06 & 0.09 & bdl & bdl & 0.05 & 0.11 & bdl & 0.14 & 0.04 \\
\hline $\mathrm{ThO}_{2}$ & 17.44 & 17.30 & 17.67 & 17.49 & 17.56 & 17.78 & 18.59 & 16.32 & 17.82 & 17.91 & 18.22 & 17.80 & 17.44 & 17.48 & 17.28 & 18.07 & 17.47 & 17.63 \\
\hline $\mathrm{UO}_{2}$ & 0.41 & 0.40 & 0.47 & 0.47 & 0.52 & 0.48 & 0.49 & 0.41 & 0.48 & 0.44 & 0.42 & 0.46 & 0.47 & 0.49 & 0.43 & 0.53 & 0.47 & 0.46 \\
\hline $\mathrm{PbO}$ & 1.01 & 1.11 & 1.02 & 1.11 & 1.05 & 1.19 & 1.17 & 1.07 & 1.01 & 1.09 & 1.06 & 1.25 & 1.07 & 0.96 & 1.06 & 1.13 & 1.14 & 1.09 \\
\hline $\begin{array}{l}\text { Total } \\
\text { apfu }\end{array}$ & 96.67 & 95.95 & 97.49 & 97.26 & 96.54 & 97.34 & 98.17 & 93.50 & 97.37 & 96.43 & 97.61 & 97.36 & 96.14 & 97.41 & 96.77 & 97.02 & 97.16 & 96.83 \\
\hline $\mathrm{La}$ & 0.059 & 0.060 & 0.061 & 0.056 & 0.060 & 0.061 & 0.057 & 0.058 & 0.057 & 0.058 & 0.057 & 0.059 & 0.059 & 0.058 & 0.060 & 0.064 & 0.061 & 0.059 \\
\hline Pr & 0.024 & 0.025 & 0.028 & 0.025 & 0.029 & 0.025 & 0.027 & 0.029 & 0.026 & 0.026 & 0.029 & 0.027 & 0.028 & 0.028 & 0.028 & 0.028 & 0.028 & 0.027 \\
\hline $\mathrm{Nd}$ & 0.104 & 0.103 & 0.106 & 0.106 & 0.105 & 0.103 & 0.107 & 0.106 & 0.105 & 0.104 & 0.104 & 0.105 & 0.106 & 0.106 & 0.103 & 0.106 & 0.103 & 0.105 \\
\hline $\mathrm{Sm}$ & 0.195 & 0.193 & 0.195 & 0.199 & 0.196 & 0.192 & 0.193 & 0.196 & 0.200 & 0.192 & 0.193 & 0.190 & 0.191 & 0.192 & 0.195 & 0.189 & 0.196 & 0.194 \\
\hline $\mathrm{Gd}$ & 0.078 & 0.076 & 0.074 & 0.077 & 0.073 & 0.077 & 0.078 & 0.077 & 0.077 & 0.074 & 0.078 & 0.077 & 0.069 & 0.076 & 0.069 & 0.072 & 0.076 & 0.075 \\
\hline $\mathrm{Tb}$ & - & - & - & - & - & - & - & - & - & - & - & 0.001 & 0.001 & - & 0.001 & - & 0.001 & 0.000 \\
\hline Dy & 0.004 & 0.004 & 0.004 & 0.004 & 0.005 & 0.004 & 0.004 & 0.005 & 0.004 & 0.005 & 0.003 & 0.005 & 0.005 & 0.005 & 0.004 & 0.003 & 0.003 & 0.004 \\
\hline Y & 0.021 & 0.017 & 0.016 & 0.016 & 0.014 & 0.011 & 0.013 & 0.009 & 0.009 & 0.015 & 0.016 & 0.009 & 0.015 & 0.017 & 0.019 & 0.013 & 0.015 & 0.014 \\
\hline $\mathrm{Ca}$ & 0.128 & 0.130 & 0.130 & 0.132 & 0.124 & 0.128 & 0.132 & 0.138 & 0.118 & 0.131 & 0.126 & 0.135 & 0.131 & 0.134 & 0.136 & 0.128 & 0.122 & 0.130 \\
\hline $\mathrm{Fe}$ & - & - & - & - & - & - & - & 0.001 & - & - & - & - & - & - & - & - & - & 0.000 \\
\hline $\mathrm{P}$ & 0.971 & 0.971 & 0.966 & 0.971 & 0.971 & 0.972 & 0.956 & 0.970 & 0.994 & 0.966 & 0.964 & 0.968 & 0.971 & 0.964 & 0.971 & 0.964 & 0.964 & 0.969 \\
\hline $\mathrm{Si}$ & 0.051 & 0.052 & 0.052 & 0.051 & 0.052 & 0.049 & 0.054 & 0.050 & 0.037 & 0.052 & 0.050 & 0.051 & 0.049 & 0.050 & 0.047 & 0.047 & 0.051 & 0.050 \\
\hline $\mathrm{S}$ & 0.001 & - & - & - & 0.001 & - & 0.005 & - & - & 0.002 & 0.003 & - & - & 0.001 & 0.003 & - & 0.004 & 0.001 \\
\hline Th & 0.162 & 0.162 & 0.164 & 0.162 & 0.161 & 0.165 & 0.172 & 0.157 & 0.159 & 0.168 & 0.169 & 0.165 & 0.164 & 0.162 & 0.160 & 0.169 & 0.163 & 0.164 \\
\hline $\mathrm{U}$ & 0.004 & 0.004 & 0.004 & 0.004 & 0.004 & 0.004 & 0.004 & 0.004 & 0.004 & 0.004 & 0.004 & 0.004 & 0.004 & 0.004 & 0.004 & 0.005 & 0.004 & 0.004 \\
\hline $\mathrm{Pb}$ & 0.011 & 0.012 & 0.011 & 0.012 & 0.013 & 0.013 & 0.013 & 0.012 & 0.011 & 0.012 & 0.012 & 0.014 & 0.012 & 0.010 & 0.012 & 0.013 & 0.013 & 0.012 \\
\hline Total & 1.993 & 1.994 & 1.997 & 1.995 & 1.992 & 1.993 & 1.996 & 2.000 & 1.981 & 1.994 & 1.993 & 1.997 & 1.995 & 1.999 & 1.995 & 1.997 & 1.992 & 1.994 \\
\hline
\end{tabular}


Table 4. Summary of chemical composition $(a p f u)$ from 17 EPMA spots for samples 2a and 4a.

\begin{tabular}{|c|c|c|c|c|c|c|c|c|c|c|c|c|c|c|c|c|c|c|}
\hline & S1 & S2 & S3 & S4 & S5 & S6 & S7 & S8 & S9 & S10 & S11 & S12 & S13 & S14 & S15 & S16 & S17 & Av \\
\hline \multicolumn{19}{|l|}{ Sample 2a } \\
\hline$\Sigma$ Ce site & 1.008 & 0.996 & 0.994 & 0.996 & 1.005 & 0.999 & 1.011 & 0.989 & 1.005 & 1.001 & 1.002 & 0.993 & 1.008 & 1.026 & 1.018 & 0.994 & 0.998 & 1.003 \\
\hline$\Sigma \mathrm{P}$ site & 0.997 & 1.005 & 1.005 & 1.006 & 0.999 & 1.003 & 0.996 & 1.009 & 0.999 & 1.002 & 1.001 & 1.004 & 0.995 & 0.983 & 0.990 & 1.004 & 1.004 & 1.000 \\
\hline$\Sigma\left[\left(\operatorname{REE}^{3+}, \mathrm{Y}^{3+}\right)+\mathrm{P}^{5+}\right]$ & 1.822 & 1.828 & 1.819 & 1.820 & 1.819 & 1.820 & 1.823 & 1.816 & 1.818 & 1.829 & 1.847 & 1.959 & 1.937 & 1.924 & 1.874 & 1.959 & 1.915 & 1.861 \\
\hline$\Sigma\left(\mathrm{Th}^{4+}+\mathrm{Si}^{4+}\right)$ & 0.166 & 0.153 & 0.161 & 0.165 & 0.169 & 0.164 & 0.167 & 0.162 & 0.168 & 0.157 & 0.139 & 0.027 & 0.048 & 0.059 & 0.116 & 0.027 & 0.060 & 0.124 \\
\hline $2 \times \Sigma\left(\operatorname{REE}^{3+}, \mathrm{Y}^{3+}\right)$ & 1.814 & 1.810 & 1.789 & 1.802 & 1.809 & 1.802 & 1.822 & 1.785 & 1.812 & 1.814 & 1.831 & 1.925 & 1.918 & 1.925 & 1.876 & 1.926 & 1.891 & 1.844 \\
\hline$\Sigma\left(\mathrm{Ca}^{2+}+\mathrm{Th}^{4+}\right)$ & 0.096 & 0.084 & 0.092 & 0.090 & 0.094 & 0.092 & 0.094 & 0.091 & 0.094 & 0.087 & 0.079 & 0.029 & 0.045 & 0.052 & 0.076 & 0.028 & 0.043 & 0.074 \\
\hline \multicolumn{19}{|l|}{ Sample 4a } \\
\hline$\Sigma$ Sm site & 0.971 & 0.971 & 0.979 & 0.973 & 0.968 & 0.972 & 0.981 & 0.979 & 0.950 & 0.975 & 0.976 & 0.978 & 0.976 & 0.984 & 0.974 & 0.985 & 0.972 & 0.974 \\
\hline$\Sigma\left[\left(\operatorname{REE}^{3+}, \mathrm{Y}^{3+}\right)+\mathrm{P}^{5+}\right]$ & 1.637 & 1.634 & 1.635 & 1.634 & 1.636 & 1.633 & 1.615 & 1.637 & 1.652 & 1.626 & 1.629 & 1.628 & 1.636 & 1.636 & 1.633 & 1.634 & 1.635 & 1.634 \\
\hline$\Sigma\left(\mathrm{Th}^{4+}+\mathrm{Si}^{4+}\right)$ & 0.213 & 0.214 & 0.216 & 0.213 & 0.214 & 0.214 & 0.226 & 0.208 & 0.195 & 0.220 & 0.219 & 0.216 & 0.212 & 0.212 & 0.207 & 0.217 & 0.213 & 0.213 \\
\hline $3 \times \mathrm{Sm}^{3+}$ & 0.584 & 0.579 & 0.584 & 0.596 & 0.588 & 0.576 & 0.578 & 0.588 & 0.600 & 0.576 & 0.579 & 0.571 & 0.574 & 0.577 & 0.585 & 0.568 & 0.589 & 0.582 \\
\hline$\Sigma\left(\mathrm{Ce}^{3+}+\mathrm{Ca}^{2+}+\mathrm{Th}^{4+}\right)$ & 0.472 & 0.477 & 0.481 & 0.473 & 0.471 & 0.483 & 0.486 & 0.482 & 0.457 & 0.486 & 0.481 & 0.488 & 0.486 & 0.487 & 0.479 & 0.491 & 0.472 & 0.480 \\
\hline
\end{tabular}

Table 5. Concentrations of $\mathrm{U}, \mathrm{Th}$, and $\mathrm{Pb}$, chemical age, and $\alpha$-radiation doses for samples $2 \mathrm{a}$ and $4 \mathrm{a}$.

\begin{tabular}{|c|c|c|c|c|c|c|c|}
\hline Sample & EPMA Spots & Th (ppm) & $\mathrm{U}(\mathrm{ppm})$ & $\mathrm{Pb}(\mathrm{ppm})$ & Age (Ma) & Average Age (Ma) & Radiation Dose ( $\alpha$-Decay Events/mg) \\
\hline \multirow{8}{*}{$2 \mathrm{a}$} & A1 & 79,664 & 2424 & 2339 & 604 & \multirow{8}{*}{$655 \pm 39$} & \multirow{8}{*}{$4.68 \times 10^{16}$} \\
\hline & $\mathrm{A} 2$ & 82,696 & 3693 & 2757 & 659 & & \\
\hline & A3 & 82,019 & 3015 & 2609 & 643 & & \\
\hline & A4 & 85,078 & 3623 & 2748 & 642 & & \\
\hline & A5 & 79,005 & 1745 & 2664 & 711 & & \\
\hline & A6 & 74,514 & 4531 & 2850 & 724 & & \\
\hline & A7 & 91,264 & 1075 & 2757 & 657 & & \\
\hline & A8 & 72,168 & 2459 & 2126 & 600 & & \\
\hline \multirow{9}{*}{$4 \mathrm{a}$} & A1 & 150,479 & 3976 & 9831 & 1360 & \multirow{9}{*}{$1361 \pm 90$} & \multirow{9}{*}{$1.93 \times 10^{17}$} \\
\hline & $\mathrm{A} 2$ & 157,430 & 3914 & 10,147 & 1348 & & \\
\hline & A3 & 160,110 & 3693 & 9831 & 1291 & & \\
\hline & A4 & 156,419 & 4020 & 11,605 & 1548 & & \\
\hline & A5 & 153,264 & 4099 & 9971 & 1353 & & \\
\hline & A6 & 153,590 & 4311 & 8866 & 1196 & & \\
\hline & A7 & 151,832 & 3746 & 9822 & 1353 & & \\
\hline & A8 & 158,827 & 4672 & 10,500 & 1364 & & \\
\hline & A9 & 153,546 & 4169 & 10,593 & 1433 & & \\
\hline
\end{tabular}




\subsection{Synchrotron High-Resolution Powder X-ray Diffraction (HRPXRD)}

Monazite crystals were hand-picked under a stereomicroscope and crushed into a fine powder $(<10 \mu \mathrm{m}$ in diameter) using an agate mortar and pestle for the HRPXRD experiment, which was conducted at beamline 11-BM, Advanced Photon Source, Argonne National Laboratory, Dupage County, IL, USA. The powdered samples were loaded into Kapton capillaries ( $0.8 \mathrm{~mm}$ internal diameter), sealed with glass wool, and rotated during the experiment at a rate of 90 rotations per second. Data were collected to a maximum $2 \theta$ of about $50^{\circ}$ with a step size of $0.001^{\circ}$ and a step time of $0.1 \mathrm{~s} / \mathrm{step}$. The HRPXRD data were collected using 12 silicon crystal analyzers that allow for high angular resolution, high precision, and accurate diffraction peak positions. A silicon (NIST 640c) and alumina (NIST 676a) standard (ratio of $1 /{ }_{3} \mathrm{Si}_{\text {to }} 2 /{ }_{3} \mathrm{Al}_{2} \mathrm{O}_{3}$ by weight) was used to calibrate the instrument and refine the monochromatic wavelength used in the experiment (Table 6). Technical aspects of the experimental set-up are given elsewhere [26-28]. The experimental techniques used in this study are well established [29-35].

Table 6. HRPXRD data and Rietveld refinement statistical indicators for samples $2 a$ and $4 a$.

\begin{tabular}{ccccc}
\hline & \multicolumn{3}{c}{ 2a } & 4a \\
\cline { 2 - 5 } & $\begin{array}{c}\text { Phase 2a } \\
\text { Monazite-(Ce) }\end{array}$ & $\begin{array}{c}\text { Phase 2b } \\
\text { Monazite-(Ce) }\end{array}$ & $\begin{array}{c}\text { Phase 2c } \\
\text { Xenotime-(Y) }\end{array}$ & Monazite-(Sm) \\
\hline Space group & $P 2_{1} / n$ & $P 2_{1} / n$ & $I 4_{1} /$ amd & $P 2_{1} / n$ \\
$a(\AA)$ & $6.8072(1)$ & $6.7551(2)$ & $6.90706(9)$ & $6.73167(6)$ \\
$b(\AA)$ & $7.00689(8)$ & $6.9804(2)$ & & $6.94489(5)$ \\
$c(\AA)$ & $6.47476(7)$ & $6.4687(1)$ & $6.0348(1)$ & $6.44964(5)$ \\
$\beta\left({ }^{\circ}\right)$ & $103.781(1)$ & $103.707(2)$ & & $103.899(1)$ \\
$V\left(\AA^{3}\right)$ & $299.940(7)$ & $296.34(1)$ & $287.906(8)$ & $292.697(4)$ \\
wt. \% & $30.5(2)$ & $66.0(2)$ & $3.5(1)$ & 100 \\
$1 \mathrm{~N}_{\text {data }}$ & 29,948 & & & 37,505 \\
$2 \mathrm{~N}_{\text {obs }}$ & 1964 & & & 1375 \\
Variables & 85 & & & 54 \\
${ }^{3}$ Overall $R\left(F^{2}\right)$ & 0.0157 & & & 0.0176 \\
$w R p$ & 0.0534 & & & 0.0539 \\
Reduced $\chi^{2}$ & 2.289 & & & 1.767 \\
$\lambda(\AA)$ & $0.45900(2)$ & & & $0.41370(2)$ \\
$2 \theta$ range & $4.5-34.5^{\circ}$ & & & $2-39.5^{\circ}$ \\
\hline
\end{tabular}

${ }^{1} \mathrm{~N}_{\text {data }}$ is the number of data points. ${ }^{2} \mathrm{~N}_{\text {obs }}$ is the number of observed reflections. ${ }^{3}$ Overall $R\left(F^{2}\right)=\left[\sum\left(F_{o}^{2}-F_{c}^{2}\right) / \sum\left(F_{o}^{2}\right)\right]^{1 / 2}$ based on observed and calculated structural amplitudes.

The HRPXRD trace for sample 2a shows broad and asymmetrical peaks indicating multiple phases and was modeled using three phases (Figure 2a). The HRPXRD trace for sample 4a shows narrow and symmetrical peaks and was modeled using a single phase (Figure 2b).

\subsection{Rietveld Structural Refinement}

The HRPXRD data for samples 2a and 4a were analyzed with the Rietveld method [36], as implemented in the GSAS program [37], and using the EXPGUI interface [38]. The initial unit-cell parameters and atom coordinates for monazite-(Ce), monazite-(Sm), and xenotime were from $\mathrm{Ni}$ et al. [11]. Scattering curves for neutral atoms were used. The background was modeled using a Chebyschev polynomial (eight terms). The peak profiles were fitted with the pseudo-Voigt function (profile type-3) in the GSAS program [39]. A full matrix leastsquares refinement was carried out by varying the parameters in the following sequence: a scale factor, unit-cell parameters, atom coordinates, and isotropic displacement parameters. HRPXRD data for sample 2a was refined using three phases: two monazite-(Ce) phases $(2 \mathrm{a}$ and $2 \mathrm{~b}$ ) and a xenotime-( $(\mathrm{Y})(=$ phase $2 \mathrm{c})$. Site occupancy factors (sofs) for Ce for phases $2 a$ and $2 b$ and $Y$ for phase $2 c$ were refined, but sofs for $P$ and $O$ were fixed to 1 . HRPXRD data for sample 4a was refined using a single phase. The sof for Sm was refined, but the 
sofs for $\mathrm{P}$ and $\mathrm{O}$ was fixed to 1 . In the final stages of the refinement, all the parameters were allowed to vary simultaneously, and the refinement converged. The fitted HRPXRD data for samples 2a and 4a are shown in Figure 2. The unit-cell parameters and the data collection and refinement statistics are given in Table 6. The atom coordinates, isotropic displacement parameters, and sofs are given in Table 7. Selected bond distances and angles are given in Table 8.
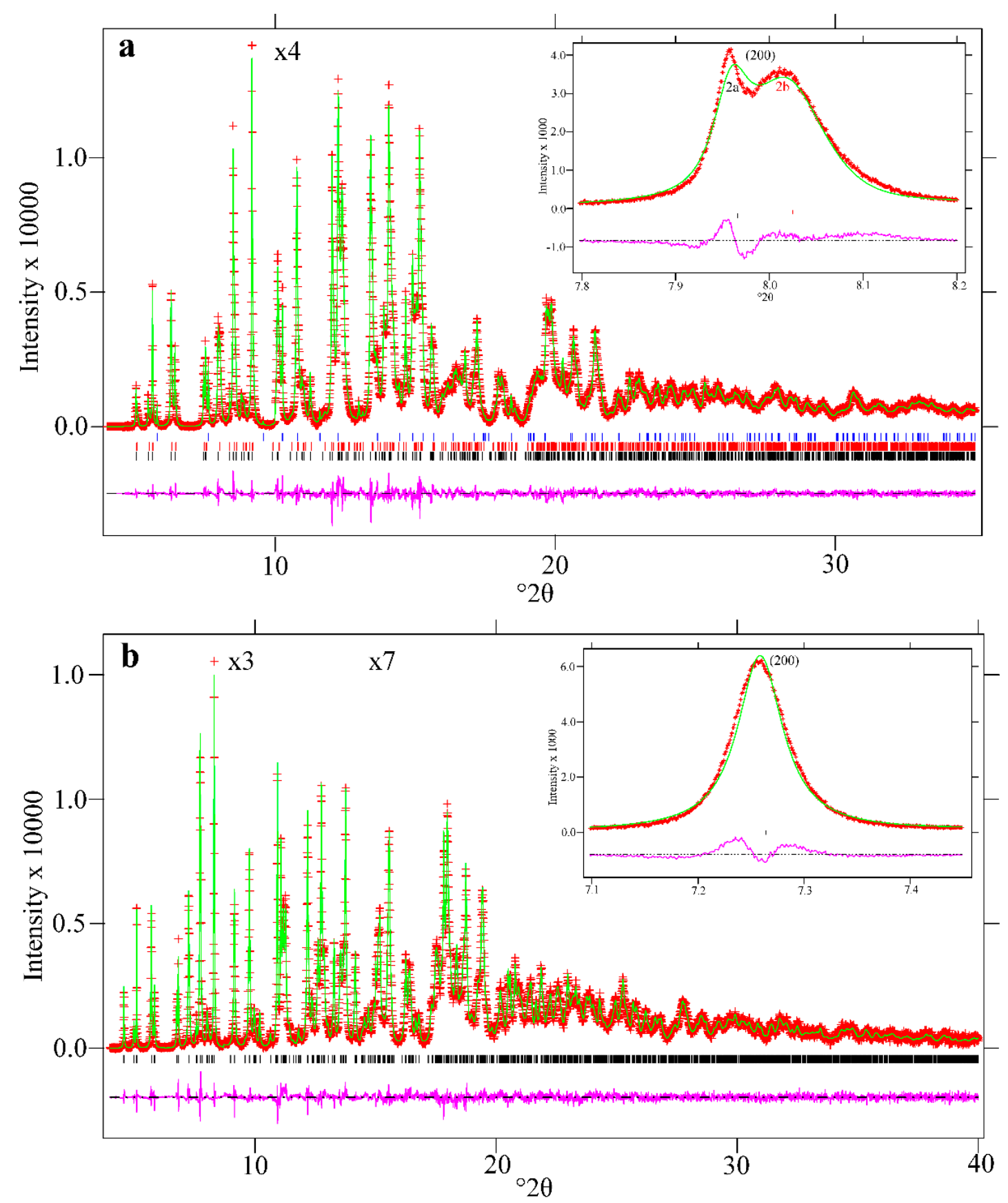

Figure 2. HRPXRD traces for (a) sample 2a and (b) sample 4a showing calculated (continuous green line) and observed (red crosses) data. The short vertical lines indicate allowed reflection positions. The intensities for the trace and difference curve in (a) that are above $10^{\circ} 2 \theta$ are multiplied by 4 . The intensities for the trace and difference curve in (b) that are above 9 and $15^{\circ} 2 \theta$ are multiplied by 3 and 7, respectively. Expanded 200 peak is shown in the inserts. The 200 peak splitting in (a) indicates the presence of two different monazite-(Ce) phases. In (a), the vertical lines for the three phases are indicated by black for phase 2a [monazite-(Ce)], red for phase 2b [monazite-(Ce)], and blue for phase 2c [xenotime-(Y)]. 
Table 7. Atom positions, isotropic displacement parameters, and site occupancy factors (sofs) for samples $2 \mathrm{a}$ and $4 \mathrm{a}$.

\begin{tabular}{|c|c|c|c|c|c|c|c|}
\hline Sample No. & Phases & Atom & Sof & $x$ & $y$ & $z$ & Uiso \\
\hline \multirow{15}{*}{$2 a$} & \multirow{6}{*}{ Phase 2a } & $\mathrm{Ce}$ & $1.120(5)$ & $0.2842(1)$ & $0.1597(1)$ & $0.0985(1)$ & $1.02(1)$ \\
\hline & & $\mathrm{P}$ & 1 & $0.3121(7)$ & $0.1623(6)$ & $0.6186(6)$ & $1.33(5)$ \\
\hline & & $\mathrm{O} 1$ & 1 & $0.2542(13)$ & $0.0113(9)$ & $0.4434(11)$ & $0.49(7)$ \\
\hline & & $\mathrm{O} 2$ & 1 & $0.3960(11)$ & $0.3297(9)$ & $0.5134(12)$ & $0.49(7)$ \\
\hline & & O3 & 1 & $0.4722(10)$ & $0.1008(11)$ & $0.8166(9)$ & $0.49(7)$ \\
\hline & & $\mathrm{O} 4$ & 1 & $0.1158(9)$ & $0.2167(10)$ & $0.6792(11)$ & $0.49(7)$ \\
\hline & \multirow{6}{*}{ Phase $2 b$} & $\mathrm{Ce}$ & $0.903(3)$ & $0.2794(1)$ & $0.1580(2)$ & $0.1013(2)$ & $1.02(1)$ \\
\hline & & $\mathrm{P}$ & 1 & $0.2913(5)$ & $0.1598(6)$ & $0.6040(5)$ & $1.33(5)$ \\
\hline & & O1 & 1 & $0.2365(11)$ & $0.0100(8)$ & $0.4262(9)$ & $0.49(7)$ \\
\hline & & $\mathrm{O} 2$ & 1 & $0.3749(8)$ & $0.3281(7)$ & $0.4968(10)$ & $0.49(7)$ \\
\hline & & $\mathrm{O} 3$ & 1 & $0.4654(7)$ & $0.1133(9)$ & $0.7964(7)$ & $0.49(7)$ \\
\hline & & $\mathrm{O} 4$ & 1 & $0.1313(8)$ & $0.2191(9)$ & $0.7227(9)$ & $0.49(7)$ \\
\hline & \multirow{3}{*}{ Phase 2c } & $Y$ & $1.252(11)$ & 0 & 0.75 & 0.125 & $1.02(1)$ \\
\hline & & $\mathrm{P}$ & 1 & 0 & 0.25 & 0.375 & $1.33(5)$ \\
\hline & & $\mathrm{O}$ & 1 & 0 & $0.0642(1)$ & $0.2369(2)$ & $0.49(7)$ \\
\hline \multirow{6}{*}{$4 a$} & & Sm & $0.912(2)$ & $0.27989(5)$ & $0.15813(5)$ & $0.10053(5)$ & $1.01(1)$ \\
\hline & & $\mathrm{P}$ & 1 & $0.3008(2)$ & $0.1628(2)$ & $0.6095(2)$ & $1.08(4)$ \\
\hline & & O1 & 1 & $0.2466(4)$ & $0.0029(3)$ & $0.4420(4)$ & $1.14(5)$ \\
\hline & & $\mathrm{O} 2$ & 1 & $0.3828(4)$ & $0.3324(3)$ & $0.5028(4)$ & $1.14(5)$ \\
\hline & & $\mathrm{O} 3$ & 1 & $0.4727(3)$ & $0.1040(4)$ & $0.8008(3)$ & $1.14(5)$ \\
\hline & & $\mathrm{O} 4$ & 1 & $0.1258(4)$ & $0.2123(4)$ & $0.7135(4)$ & $1.14(5)$ \\
\hline
\end{tabular}

Table 8. Bond distances * $(\AA)$ and angles $\left(^{\circ}\right)$ for samples 2 a and 4 a.

\begin{tabular}{|c|c|c|c|c|c|c|}
\hline \multicolumn{5}{|c|}{$2 a$} & \multicolumn{2}{|c|}{$4 a$} \\
\hline & $\begin{array}{c}\text { Phase-2a } \\
\text { Monazite-(Ce) }\end{array}$ & $\begin{array}{c}\text { Phase-2b } \\
\text { Monazite-(Ce) }\end{array}$ & \multicolumn{2}{|c|}{$\begin{array}{c}\text { Phase-2c } \\
\text { Xenotime-(Y) }\end{array}$} & Monazite-(Sm) & \\
\hline $\mathrm{Ce}-\mathrm{O} 1^{\prime}$ & $2.515(8)$ & $2.420(6)$ & $\mathrm{Y}-\mathrm{O}^{\prime} \times 4$ & $2.273(1)$ & $\mathrm{Sm}-\mathrm{O} 1^{\prime}$ & $2.510(3)$ \\
\hline $\mathrm{Ce}-\mathrm{O} 1^{\prime \prime}$ & $2.486(5)$ & $2.464(5)$ & $\mathrm{Y}-\mathrm{O}^{\prime \prime} \times 4$ & $2.533(1)$ & $\mathrm{Sm}-\mathrm{O} 1^{\prime \prime}$ & $2.411(2)$ \\
\hline $\mathrm{Ce}-\mathrm{O} 2^{\prime}$ & $2.870(8)$ & $2.755(7)$ & & & $\mathrm{Sm}-\mathrm{O} 2^{\prime}$ & $2.795(3)$ \\
\hline $\mathrm{Ce}-\mathrm{O} 2^{\prime \prime}$ & $2.637(7)$ & $2.545(5)$ & & & $\mathrm{Sm}-\mathrm{O} 2^{\prime \prime}$ & $2.532(2)$ \\
\hline $\mathrm{Ce}-\mathrm{O} 2^{\prime \prime \prime}$ & $2.569(7)$ & $2.657(5)$ & & & $\mathrm{Sm}-\mathrm{O} 2^{\prime \prime \prime}$ & $2.596(2)$ \\
\hline $\mathrm{Ce}-\mathrm{O}^{\prime}$ & $2.501(8)$ & $2.596(5)$ & & & $\mathrm{Sm}-\mathrm{O} 3^{\prime}$ & $2.602(2)$ \\
\hline $\mathrm{Ce}-\mathrm{O}^{\prime \prime}$ & $2.439(6)$ & $2.541(5)$ & & & $\mathrm{Sm}-\mathrm{O}^{\prime \prime}$ & $2.446(2)$ \\
\hline $\mathrm{Ce}-\mathrm{O} 4^{\prime}$ & $2.713(7)$ & $2.453(6)$ & & & $\mathrm{Sm}-\mathrm{O} 4^{\prime}$ & $2.491(3)$ \\
\hline $\mathrm{Ce}-\mathrm{O} 4^{\prime \prime}$ & $2.357(5)$ & $2.473(5)$ & & & Sm-O4" & $2.439(2)$ \\
\hline$<\mathrm{Ce}-\mathrm{O}>$ [9] & $2.565(7)$ & $2.545(4)$ & $<\mathrm{Y}-\mathrm{O}>[8]$ & $2.403(1)$ & $<\mathrm{Sm}-\mathrm{O}>$ [9] & $2.536(2)$ \\
\hline Ce- $\mathrm{P}^{\prime}$ & $3.158(4)$ & $3.237(4)$ & $\mathrm{Y}-\mathrm{P}$ & $3.0174(1)$ & $\mathrm{Sm}-\mathrm{P}^{\prime}$ & $3.204(1)$ \\
\hline $\mathrm{Ce}-\mathrm{P}^{\prime \prime}$ & $3.327(4)$ & $3.234(4)$ & & & $\mathrm{Sm}-\mathrm{P}^{\prime \prime}$ & $3.252(1)$ \\
\hline P-O1 & $1.533(2)$ & $1.533(2)$ & & & P-O1 & $1.5316(7)$ \\
\hline $\mathrm{P}-\mathrm{O} 2$ & $1.533(2)$ & $1.538(2)$ & & & $\mathrm{P}-\mathrm{O} 2$ & $1.5319(7)$ \\
\hline P-O3 & $1.533(2)$ & $1.531(2)$ & & & P-O3 & $1.5304(6)$ \\
\hline P-O4 & $1.528(2)$ & $1.524(2)$ & & & P-O4 & $1.5282(6)$ \\
\hline$<\mathrm{P}-\mathrm{O}>[4]$ & $1.532(2)$ & $1.532(2)$ & $\mathrm{P}-\mathrm{O} \times 4$ & $1.530(1)$ & $<\mathrm{P}-\mathrm{O}>[4]$ & $1.5305(7)$ \\
\hline O1-P-O2 & $104.9(5)$ & $103.4(4)$ & $\mathrm{O}-\mathrm{P}-\mathrm{O}^{\prime} \times 4$ & $107.25(4)$ & O1-P-O2 & 106.9(2) \\
\hline O1-P-O3 & $115.5(5)$ & $117.9(5)$ & $\mathrm{O}-\mathrm{P}-\mathrm{O}^{\prime \prime} \times 2$ & 114.01(9) & O1-P-O3 & $112.4(2)$ \\
\hline O1-P-O4 & $106.0(5)$ & 119.3(4) & $<\mathrm{O}-\mathrm{P}-\mathrm{O}>[6]$ & $109.50(6)$ & O1-P-O4 & 113.2(2) \\
\hline $\mathrm{O} 2-\mathrm{P}-\mathrm{O} 3$ & $108.7(5)$ & $103.6(3)$ & & & O2-P-O3 & $106.4(2)$ \\
\hline O2-P-O4 & 111.4(5) & $113.5(5)$ & & & O2-P-O4 & $115.0(2)$ \\
\hline O3-P-O4 & $110.2(5)$ & $98.5(4)$ & & & O3-P-O4 & 102.7(2) \\
\hline$<\mathrm{O}-\mathrm{P}-\mathrm{O}>[6]$ & 109.5(5) & 109.4(4) & & & $<\mathrm{O}-\mathrm{P}-\mathrm{O}>[6]$ & $109.5(2)$ \\
\hline
\end{tabular}

* Based on SCXRD, bond valence sums for these same samples are given in Reference [5]. 


\section{Results}

\subsection{Cation Exchange in Th-Bearing Monazite-(Ce) and Monazite-(Sm)}

Two main substitution mechanisms $\left[\left(\mathrm{REE}^{3+}, \mathrm{Y}^{3+}\right)+\mathrm{P}^{5+} \leftrightarrow \mathrm{Th}^{4+}+\mathrm{Si}^{4+}\right.$ (huttonite) and $2\left(\operatorname{REE}^{3+}, \mathrm{Y}^{3+}\right) \leftrightarrow(\mathrm{Th}, \mathrm{U})^{4+}+\mathrm{Ca}^{2+}$ (cheralite)] are commonly observed in monazite [7,40-42]. Cheralite substitution dominates in metamorphic monazite, whereas the huttonite substitution is more common in granitic monazite [7,43]. Based on data given in Table 4, Figure 3a,b show the huttonite and cheralite substitutions in sample 2a. Sample 4a shows the huttonite substitution (Figure 4a), but the cheralite substitution is not evident. However, when the $\left(3 \times \mathrm{Sm}^{3+}\right)$ is plotted against the sum of $\left(\mathrm{Th}^{4+}+\mathrm{Ca}^{2+}+\mathrm{Ce}^{3+}\right)$, a linear relation is obtained (Figure $4 b$ ).
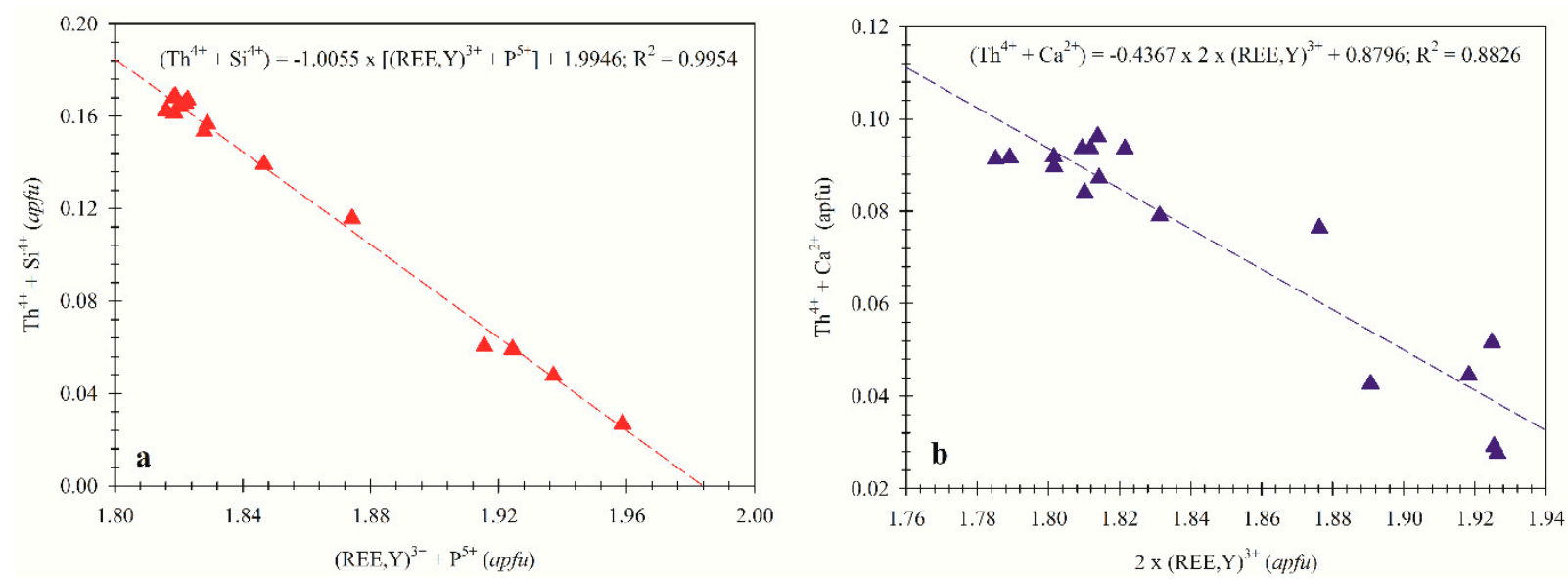

Figure 3. Compositional exchanges in sample 2a. Two exchanges are shown: (a) $(\mathrm{REE}, \mathrm{Y})^{3+}+\mathrm{P}^{5+} \leftrightarrow \mathrm{Th}^{4+}+\mathrm{Si}^{4+}($ huttonite substitution) and (b) $2(\mathrm{REE}, \mathrm{Y})^{3+} \leftrightarrow \mathrm{Th}^{4+}+\mathrm{Ca}^{2+}$ (cheralite substitution).
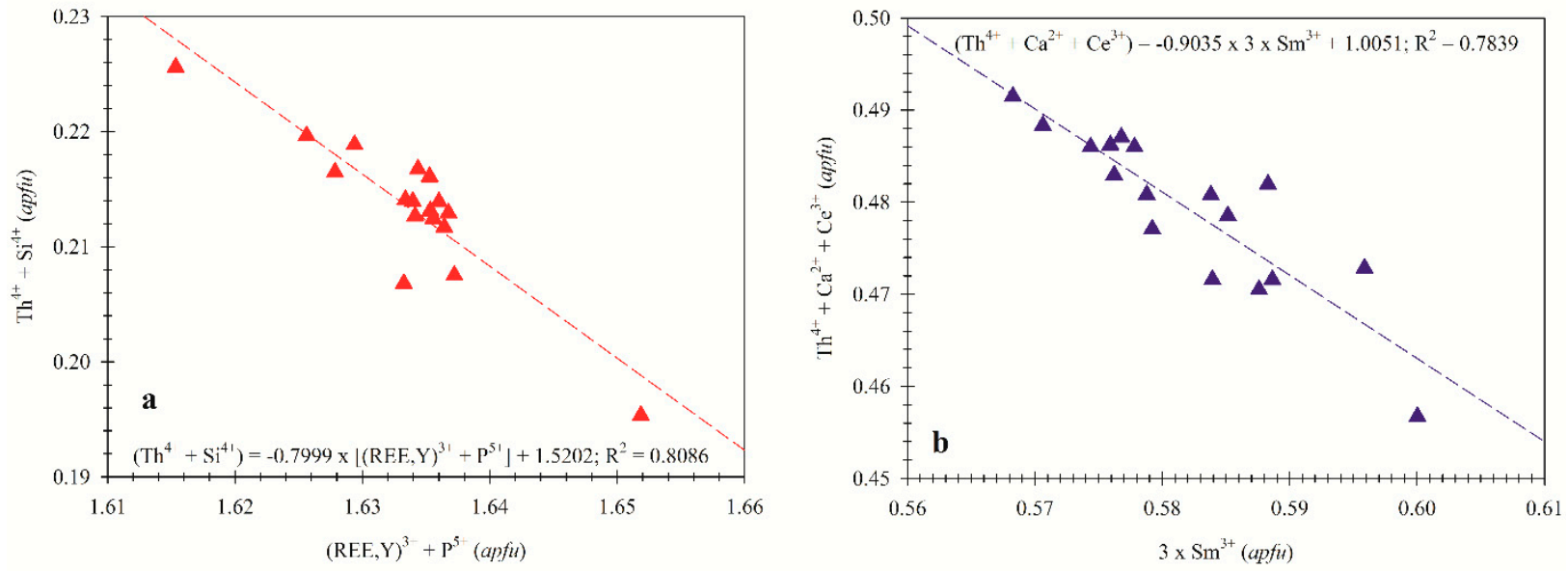

Figure 4. Compositional exchanges in sample 4a. Two negative exchanges are observed: (a) $(\mathrm{REE}, \mathrm{Y})^{3+}+\mathrm{P}^{5+} \leftrightarrow \mathrm{Th}^{4+}+\mathrm{Si}^{4+}$ (huttonite substitution) and (b) $3 \mathrm{Sm}^{3+} \leftrightarrow \mathrm{Ce}^{3+}+\mathrm{Th}^{4+}+\mathrm{Ca}^{2+}$.

\subsection{Two Monazite-(Ce) Phases and One Xenotime-(Y) in Sample $2 a$}

The synchrotron HRPXRD data for sample 2a indicates three different phases, which are monazite-(Ce) (phase $2 \mathrm{a}=30.5 \mathrm{wt} . \%$ ), monazite-(Ce) (phase $2 \mathrm{~b}=66.0 \mathrm{wt} . \%$ ), and xenotime- $(\mathrm{Y})$ (phase $2 \mathrm{c}=3.5 \mathrm{wt}$. \%). The crystal structures of the three different phases are modeled quite well, as indicated by the overall $\mathrm{R}_{\mathrm{F}}{ }^{2}$ Rietveld refinement index of 0.0157 (Table 6). Splitting of the monazite 200 peak is clearly shown in Figure 5, as well as in the inserts of Figure 2a. Broadening of the peak bases is observed for all the peaks because of the presence of two slightly different monazite phases. This peak broadening is clear 
for reflection 200, indicating significant structural changes along the a direction. The existence of two monazite phases in a crystal was reported, but it was not structurally evaluated $[15,19]$, as no phase fractions or bond distances were given. One monazite phase disappeared after heating to $1000{ }^{\circ} \mathrm{C}$ [12]. Three reflections are shown in Figure 5a for xenotime-(Y): 101, 200, and 211 reflections. Multiple phases in minerals are quite common [44-53].

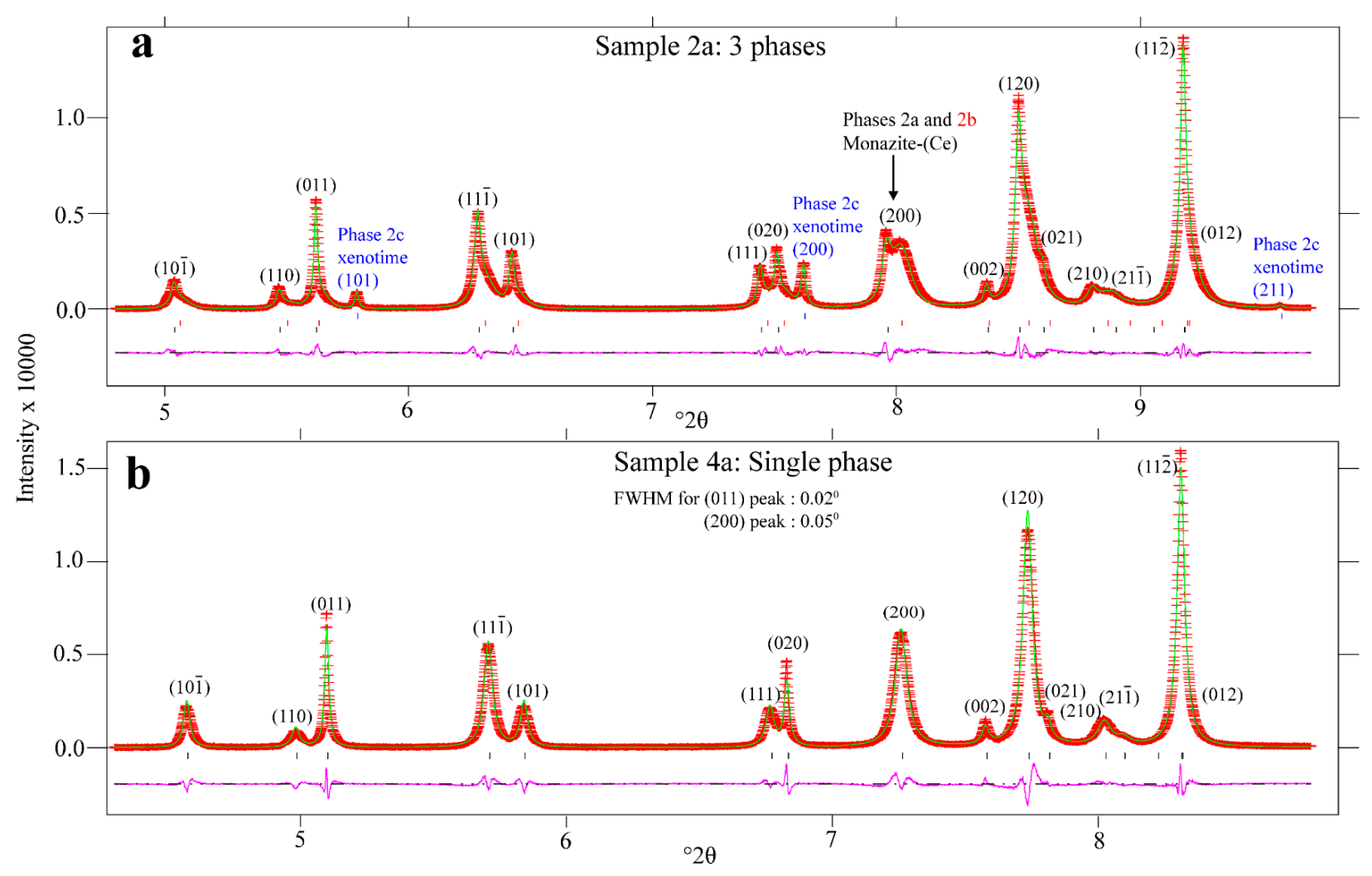

Figure 5. Expanded parts of the synchrotron HRPXRD traces. (a) Sample 2a contains three different phases: a xenotime(Y) (3.5 wt. \%) and two monazite-(Ce) phases where phase $2 \mathrm{a}$ is $30.5 \mathrm{wt}$. \% and phase $2 \mathrm{~b}$ is $66.0 \mathrm{wt}$. \%. Three peaks from xenotime-(Y) are labelled. The split 200 reflection corresponds to phases $2 \mathrm{a}$ and $2 \mathrm{~b}$. (b) Sample $4 \mathrm{a}$ is a single monazite-(Sm) phase.

The unit-cell volume for phase $2 \mathrm{a}\left[V=299.940(7) \AA^{3}\right]$ is $1.19 \%$ larger than that for phase $2 b\left[296.34(1) \AA^{3}\right]$ (Table 6). The phase $2 \mathrm{c}$ is xenotime-(Y), which was refined with space group $I 4_{1}$ / amd and has a unit-cell volume of $287.906(8) \AA^{3}$, which is $0.52 \%$ larger than previously reported [11]. The fractions of phase $2 a$ [monazite-(Ce)], phase $2 b$ [monazite(Ce)], and phase 2c [xenotime-(Y)] are 30.5(2), 66.0(2), and 3.5(1) wt. \%, respectively (Table 6).

The $a, b$, and $c$ unit-cell parameters for phase $2 \mathrm{a}$ are larger than that for phase $2 \mathrm{~b}$ (Table 6). Therefore, the difference is more prominent in the $a$ unit-cell parameter followed by the $b$ and $c$ unit-cell parameters. However, the unit-cell parameters for the dominant phase $2 b$ are closer to the values obtained with SCXRD for the same sample [5].

\subsection{Sample 4a: Monazite-(Sm)}

Sample 4a contains a single phase of monazite-(Sm). Peaks in the HRPXRD trace are symmetric and contain no peak splitting or abnormal broadenings at the peak bases (Figure 5b). The unit-cell volume for sample $4 a\left[V=292.697(4) \AA^{3}\right]$ is $0.31 \%$ smaller than that for monazite-(Sm) $\left[V=293.6(1) \AA^{3}\right]$ obtained with PXRD [54]. The Sm concentrations for sample 4a and for monazite-(Sm) are 0.194 and 0.197 apfu, respectively [54]. However, sample 4a contains a significantly lower amount of $\mathrm{Gd}$ and high quantities of $\mathrm{Ce}, \mathrm{Th}, \mathrm{Ca}$, 
$\mathrm{Nd}$, and La apfu compared to monazite-(Sm) [54] (Figure 6). The difference in chemical compositions between sample $4 \mathrm{a}$ and monazite-(Sm) [54] may contribute to the small differences in the unit-cell parameters.

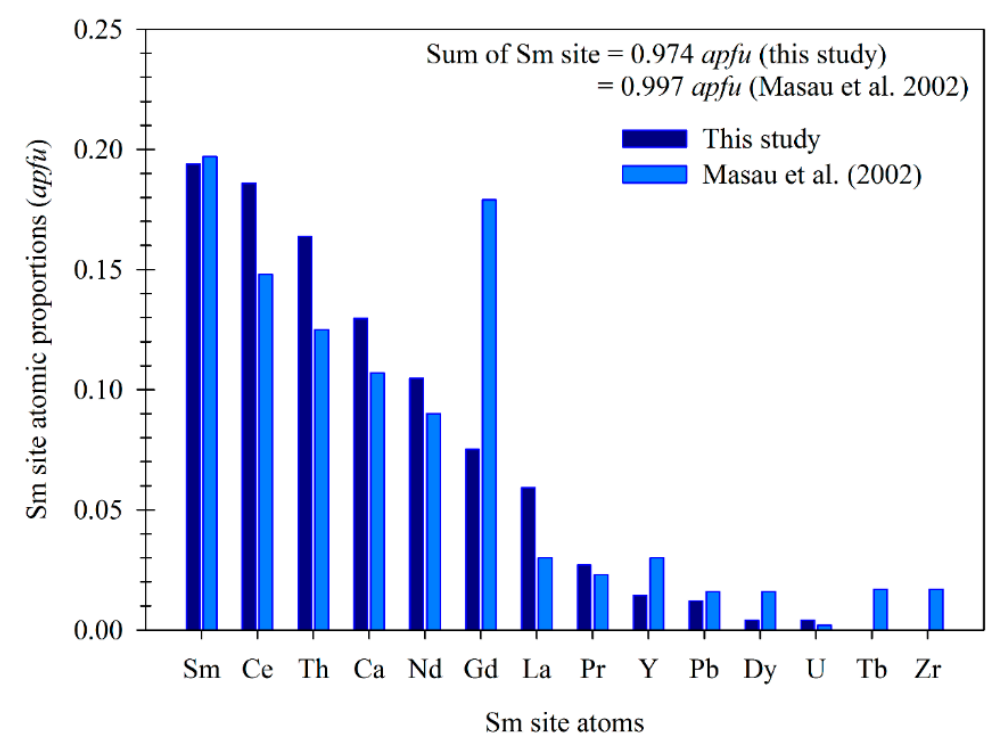

Figure 6. Atoms at the Sm site in sample 4a [monazite-(Sm)] and monazite-(Sm) in Masau et al. [54]. The main difference is in the amount of Gd atoms in the two samples.

The HRPXRD data for sample 4a is modeled very well with the Rietveld method because the overall $R_{\mathrm{F}}{ }^{2}$ value is 0.0176 (Table 6). However, the peaks are not consistent in terms of their FWHM values (Figure 5b). For example, the 200 peak has higher FWHM than the 020 and 011 peaks (Figure 5). After the refinement of the profile-3 LY (Lorentzian isotropic strain broadening) coefficient, the value is relatively high for sample 2a (LY values for phase $2 \mathrm{a}$ is 36.3 , phase $2 \mathrm{~b}$ is 87.7 , phase $3 \mathrm{c}$ is 1.3 , and sample $4 \mathrm{a}$ is 3.3$)$. For profile-3, using the isotropic LY term, the isotropic strain $(\%)=100 \% \times \operatorname{LY} \times(\pi / 18,000)$. So, the isotropic strain (\%) for phase $2 \mathrm{a}$ is 6.4 , phase $2 \mathrm{~b}$ is 15.4 , phase $2 \mathrm{c}$ is 0.2 and sample $4 \mathrm{a}$ is 0.6 . Peak broadening in the X-ray diffraction is the result of one or more of the following sources: instrumental, crystallite size, and the presence of a micro-strain [55]. The instrumental broadening is not expected because this study used synchrotron data. The large LY value is related to strain. Because of this strain, the SCXRD data for the same sample (2a here $=2$ in [5]), which has a relatively lower resolution, gave very high mosaicity and $R_{\text {int }}$ [5]. The source of this strain could be the remnant of radiation damage and accumulation. Since sample 4a contains a very high amount of Th and is relatively older (1361 Ma) in age, it received a large number of $\alpha$-radiation doses $\left(1.93 \times 10^{17} \alpha\right.$-decay events $\left./ \mathrm{mg}\right)$ (Table 5). The recovery of radiation damage is much faster in monazite relative to zircon. Since this monazite- $(\mathrm{Sm})$ received high $\alpha$-radiation doses, the damage overcame the recovery and gave rise to remnant damage in sample 4 a.

\subsection{Variations of Unit-Cell Parameters}

The unit-cell parameters for samples $2 a$ and 4 a compare well with other published data (Figure 7). Sample 2 is based on SCXRD data [5], whereas 2a, 2b, and 2c are for the same sample 2, but these are based on HRPXRD data. Again, sample 4 is based on SCXRD data [5], whereas sample $4 \mathrm{a}$ is for the same sample 4 but based on HRPXRD data. The slopes of the linear equations indicate that the changes are the highest for the $a$ and $b$ unit-cell parameters followed by the $c$ unit-cell parameters (Figure 7). The $a$ and $b$ unit-cell parameters from literature fall close to the linear regression lines, but the $c$ parameters are relatively scattered. The unit-cell parameters for Ce-dominated detrital monazite samples 1 and 3 were obtained with SCXRD. Both samples are crystalline [5]. 

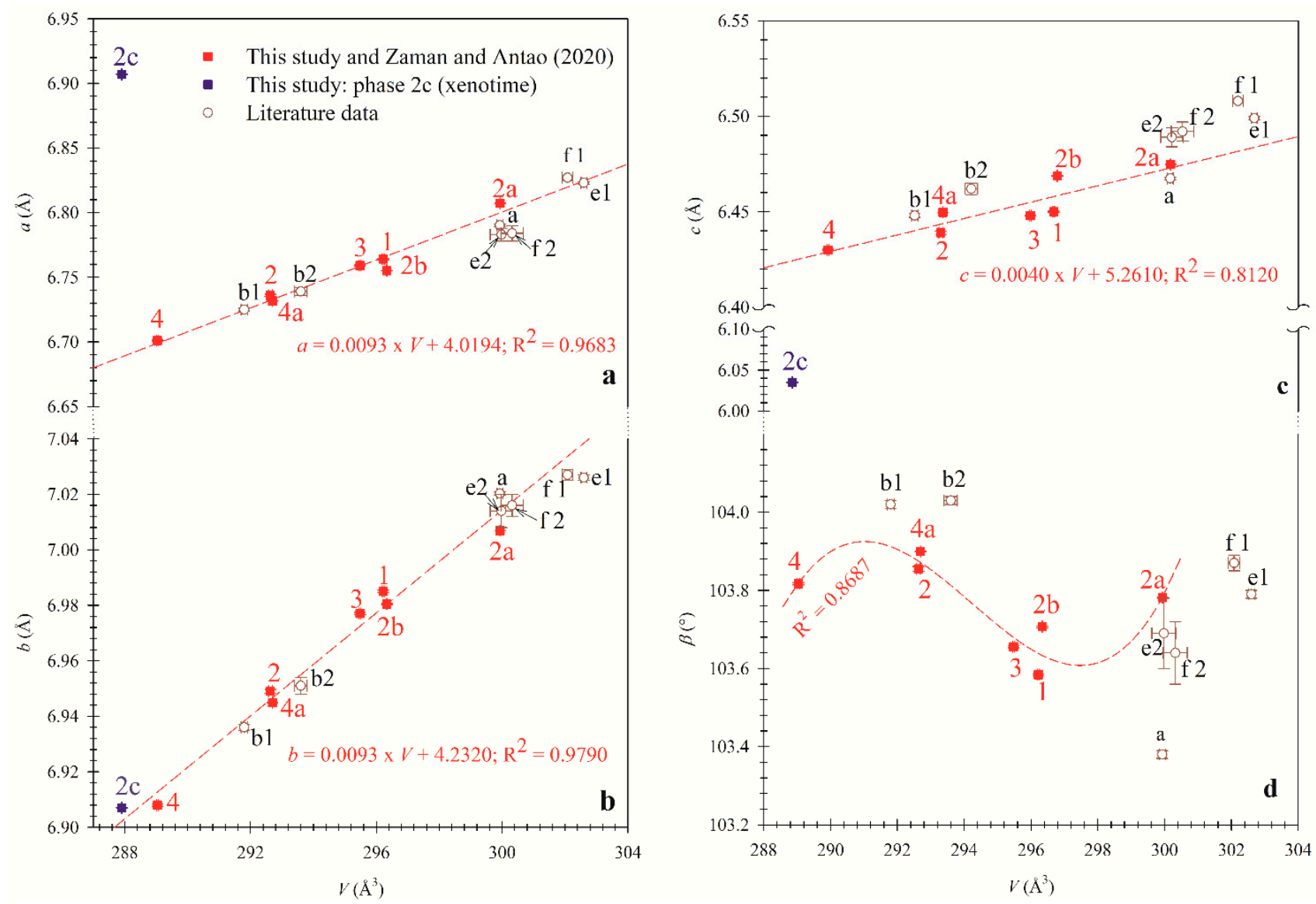

Figure 7. Variations of unit-cell parameters in monazite: (a) $a$ vs. $V,($ b) $b$ vs. $V$, (c) $c$ vs. $V$, and (d) $\beta$ vs. $V$. The dashed lines represent regression fits for our samples. Phase $2 c$ data is excluded from the fits. Open symbols are data from the literature [a: monazite-(Ce) [11], b1 (SCXRD), and b2 (PXRD) for monazite-(Sm) [54], e1 (phase 1) and e2 (phase 2) for monazite-(Ce) [15], f1 (phase 1) and f2 (phase 2) for monazite-(Ce) [19]]. Some errors are smaller than the symbols. The $a, b$, and $c$ unit-cell parameters vary with linearity with $V$, but not with the $\beta$ angle.

The $a$ and $c$ unit-cell parameters for phase $2 c$ are off the linear regression lines because xenotime- $(\mathrm{Y})$ is tetragonal with space group $14_{1} /$ amd. However, the $b(=a)$ parameter for xenotime phase $2 \mathrm{c}$ falls on the linear regression line (Figure $7 \mathrm{~b}$ ). This indicates that, during the monazite to xenotime phase transition, major changes occurred along the $\mathbf{a}$ and c directions.

\subsection{Bond Distances}

The bond distances for Th-free monazite-(Ce) were previously obtained [11]. Average $\langle\mathrm{Ce} / \mathrm{Sm}-\mathrm{O}\rangle,\langle\mathrm{Y}-\mathrm{O}\rangle$, and $\langle\mathrm{P}-\mathrm{O}\rangle$ distances are plotted with the $V$ (Figure 8 ). The average $\langle\mathrm{Y}-\mathrm{O}\rangle$ distance for phase $2 \mathrm{c}$ [xenotime- $(\mathrm{Y})]$ is off the trendline. The average $<\mathrm{Ce} / \mathrm{Sm} / \mathrm{Y}-\mathrm{O}>$ for phases $2 \mathrm{~b}$ and $2 \mathrm{c}$ are very different. Based on the following radii [56]: [9] $\mathrm{Ce}^{3+}=1.196,[8] \mathrm{Y}^{3+}=1.019$, and $[3] \mathrm{O}^{2-}=1.36, \mathrm{Ce}^{3+}-\mathrm{O}=2.556$ and $\mathrm{Y}^{3+}-\mathrm{O}=2.379 \AA$, compared to 2.565 (phase 2a) and $2.403 \AA$ (phase 2c) (Table 8). The average $\langle\mathrm{P}-\mathrm{O}\rangle$ distance is nearly constant and is about $1.528 \AA$ [5]. Metamorphic processes may trigger a monazite to xenotime phase transition [7,57]. However, sample $2 \mathrm{a}$ is of pegmatitic origin and contains a significant amount of radioactive elements, so a radiation-induced phase transition may be involved, but other chemical processes cannot be ruled out. 


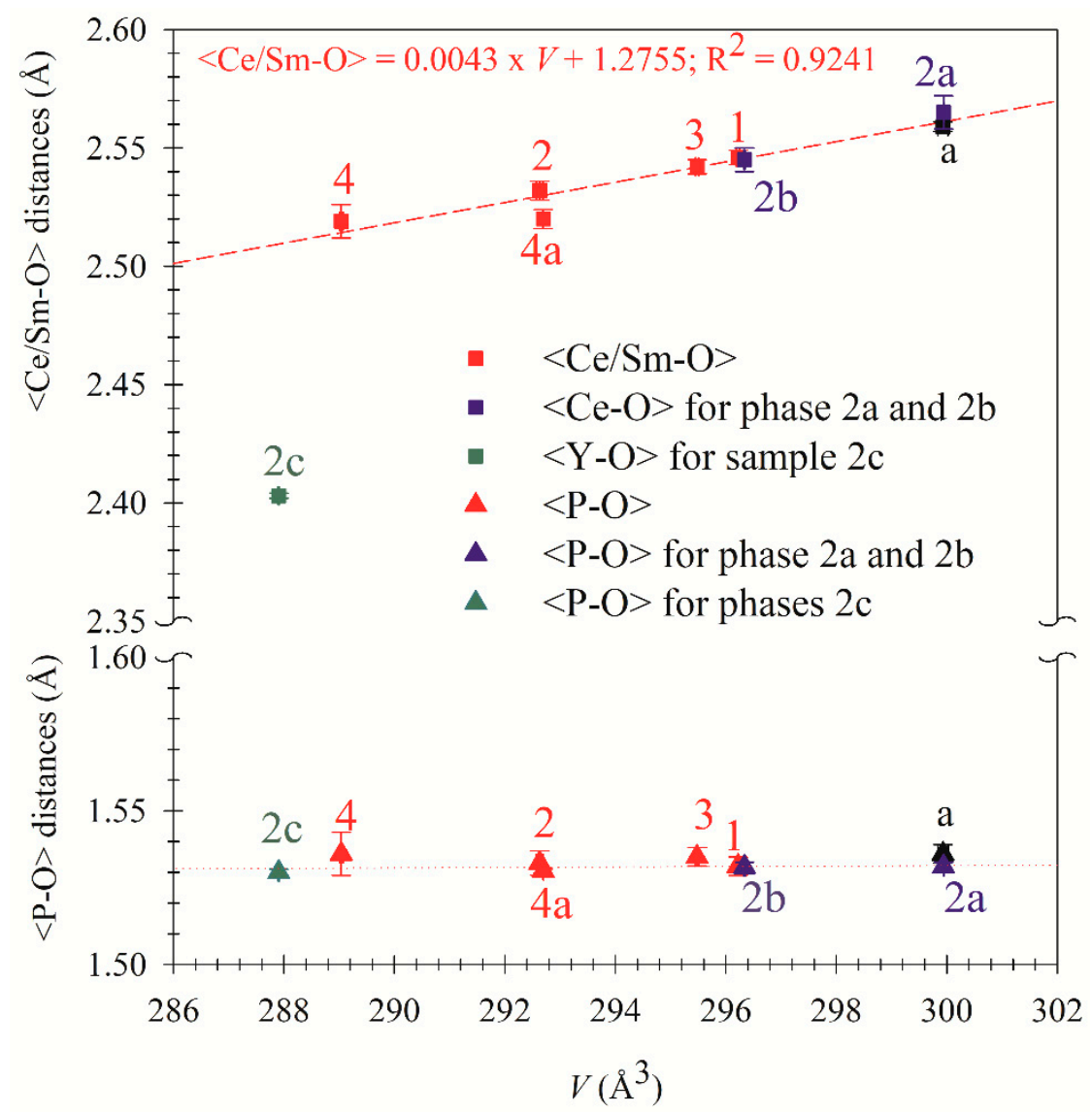

Figure 8. Variations of average $\langle\mathrm{Ce} / \mathrm{Sm}-\mathrm{O}\rangle,\langle\mathrm{Y}-\mathrm{O}\rangle$, and $\langle\mathrm{P}-\mathrm{O}\rangle$ distances with $V$. The dashed line is a linear fit to the average $<\mathrm{Ce} / \mathrm{Sm}-\mathrm{O}>$ distances including that of sample "a" from Ni et al. [11] and the equation for this line is given (insert). The dotted line is for the average $<\mathrm{P}-\mathrm{O}>$ distances. Sample 2 is based on SCXRD data [5], whereas $2 a, 2 b$, and $2 c$ are for the same sample 2 but these are based on HRPXRD data. Again, sample 4 is based on SCXRD data [5], whereas sample 4a is for the same sample 4 but based on HRPXRD data. Samples 1 and 3 were obtained with SCXRD [5].

\subsection{Ce Site Cation Distribution in Sample $2 a$}

The range of variations of oxides in sample $2 \mathrm{a}$ is higher than that in sample $4 \mathrm{a}$ (Tables 2 and 3). EPMA chemical data for sample $2 a$ indicates that some cations such as $\mathrm{Y}^{3+}, \mathrm{Ce}^{3+}, \mathrm{La}^{3+}, \mathrm{Th}^{4+}$, and $\mathrm{Si}^{4+}$ vary anomalously. Sample 2 a contains xenotime-( $\left.\mathrm{Y}\right)$ as a third phase, so the variations of $\mathrm{Ce}^{3+}, \mathrm{La}^{3+}, \mathrm{Pr}^{3+}, \mathrm{Nd}^{3+}, \mathrm{Sm}^{3+}, \mathrm{Gd}^{3+}, \mathrm{Dy}^{3+}, \mathrm{Ca}^{2+}$, and $\mathrm{Th}^{4+}$ with $\mathrm{Y}^{3+}$ concentrations are examined (Figure 9). When the concentration of $\mathrm{Y}^{3+}$ decreases, the concentrations of $\mathrm{Ce}^{3+}, \mathrm{Nd}^{3+}, \mathrm{La}^{3+}, \mathrm{Pr}^{3+}$, and $\mathrm{Sm}^{3+}$ increase (Figure 9 left). The changing slopes are $\mathrm{Ce}^{3+}>\mathrm{Nd}^{3+}>\mathrm{La}^{3+}>\mathrm{Pr}^{3+}>\mathrm{Sm}^{3+}$. In contrast, the concentrations of $\mathrm{Th}^{4+}, \mathrm{Dy}^{3+}, \mathrm{Gd}^{3+}$, and $\mathrm{Si}^{4+}$ increase with a growing concentration of $\mathrm{Y}^{3+}$ (Figure 9 right). Thus, the chemical composition also indicates that the formation of phases $2 \mathrm{a}$ and $2 \mathrm{c}$ may be related to the redistributions of cations in sample 2a. EPMA data for sample $4 a$ do not carry any distinct chemical variability, as found in sample 2 a.

The back-scattered electron (BSE) image of sample 2 a shows variations in colour and brightness that indicate chemical heterogeneity (Figure 10). The dark gray and less bright part of the crystal (labelled $x t$ ) is $Y$ and $P$ rich and Th depleted and corresponds to xenotime(Y) (Figures 10 and 11). The light gray and brightest parts (tr) are Th-rich and Si-rich and Y and REE depleted (Figures 10 and $11 \mathrm{~b}, \mathrm{c}$ ). The medium dark part and brighter part (mz) is REE-rich and P-rich domains (Figure 11a). EPMA data spots were selected only from the $\mathrm{mz}$ part of sample $2 \mathrm{a}$ to measure the chemical composition quantitatively. Fragments of sample 2a used for synchrotron HRPXRD were examined with a polarizing microscope and no twin, cracks, or anomalous birefringence were observed. One of the fragments 
was also studied with SCXRD and is modeled structurally using a single phase with good refinement statistics [5]. Therefore, the fragments used for HRPXRD data collected were from the medium dark and brighter areas (Figure 10). The HRPXRD data shows that sample 2a contains three phases: two monazite-(Ce) phases and a xenotime-Y phase.
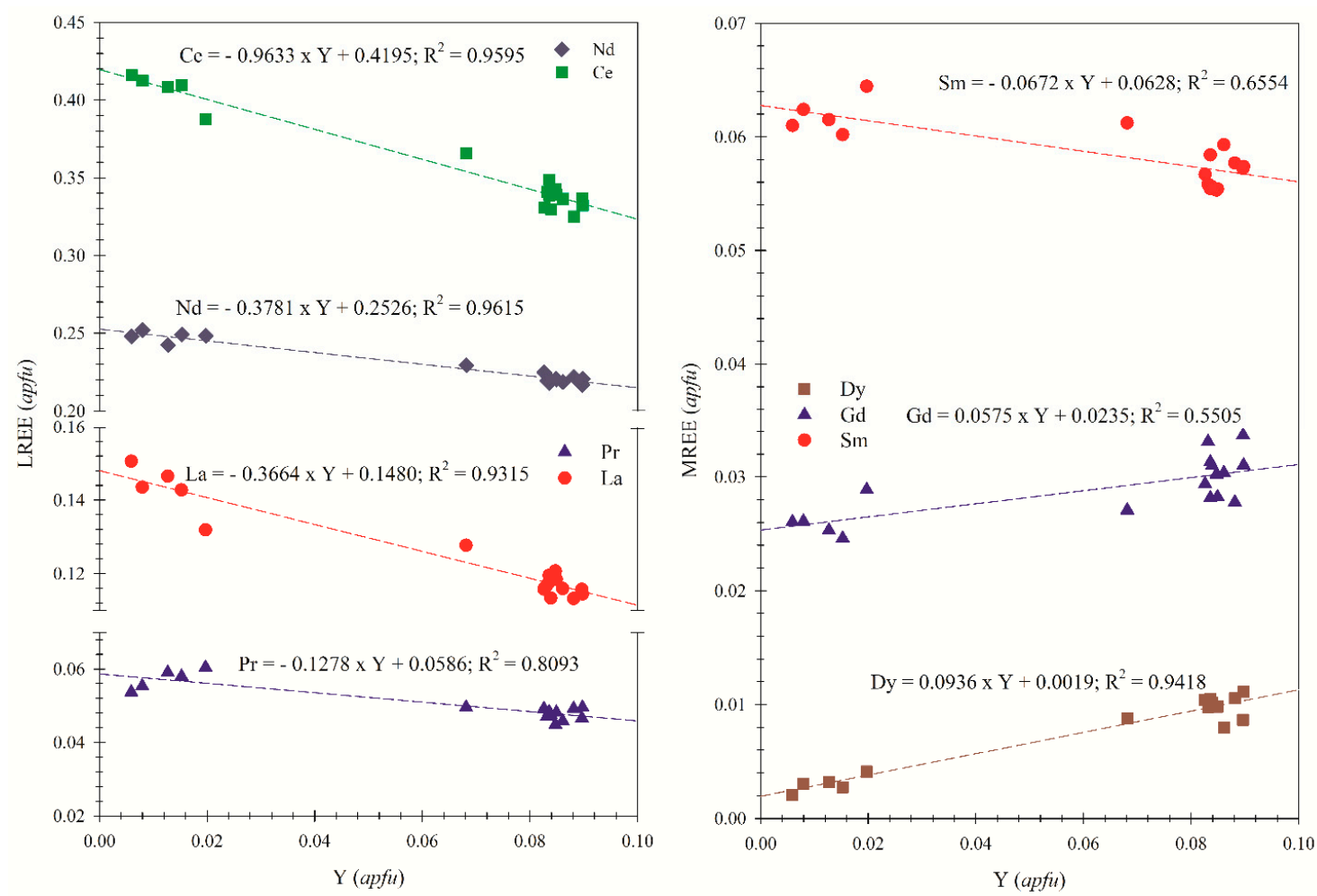

Figure 9. Variations of LREE (left set) and MREE (right set) with Y in sample 2a. The dashed lines are linear fits and their equations are given as inserts.

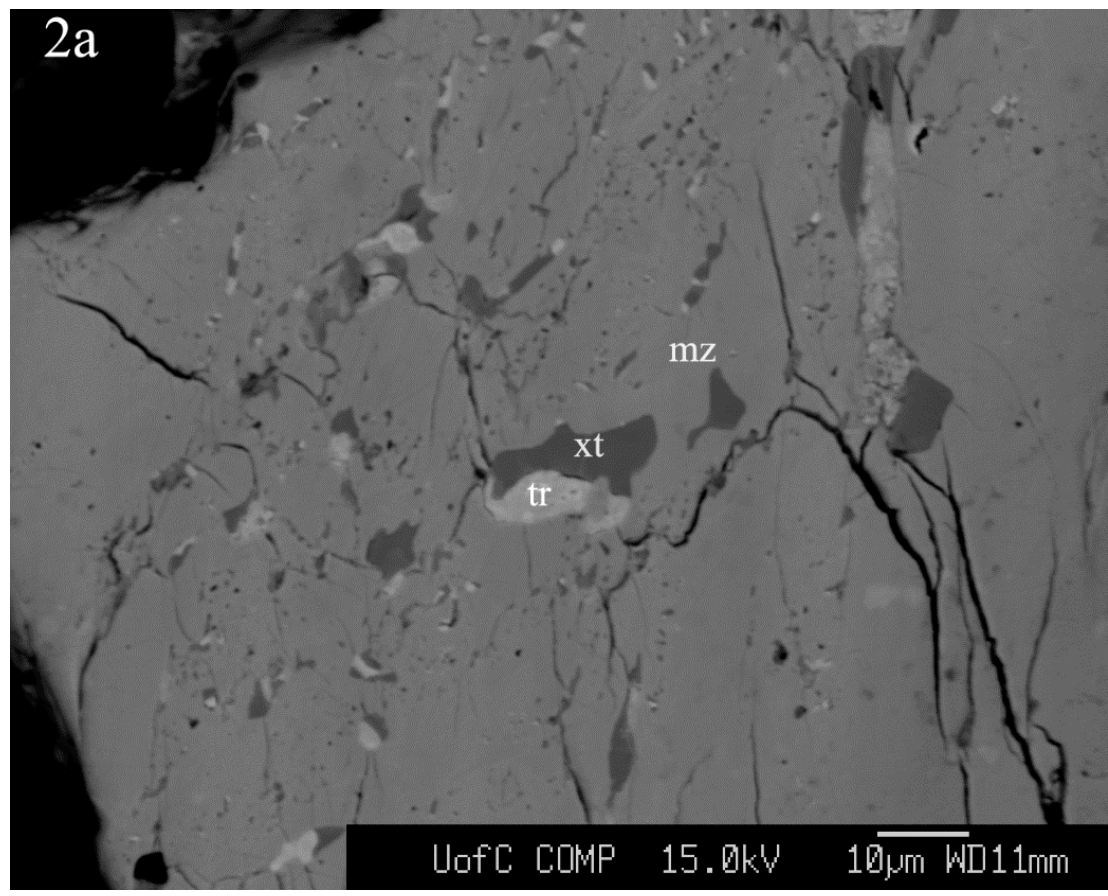

Figure 10. Back-scattered electron (BSE) image of sample 2a shows chemical heterogeneity: $\operatorname{tr}=$ thorite (light gray), $\mathrm{mz}=$ monazite (gray), $\mathrm{xt}=$ xenotime (dark gray). 

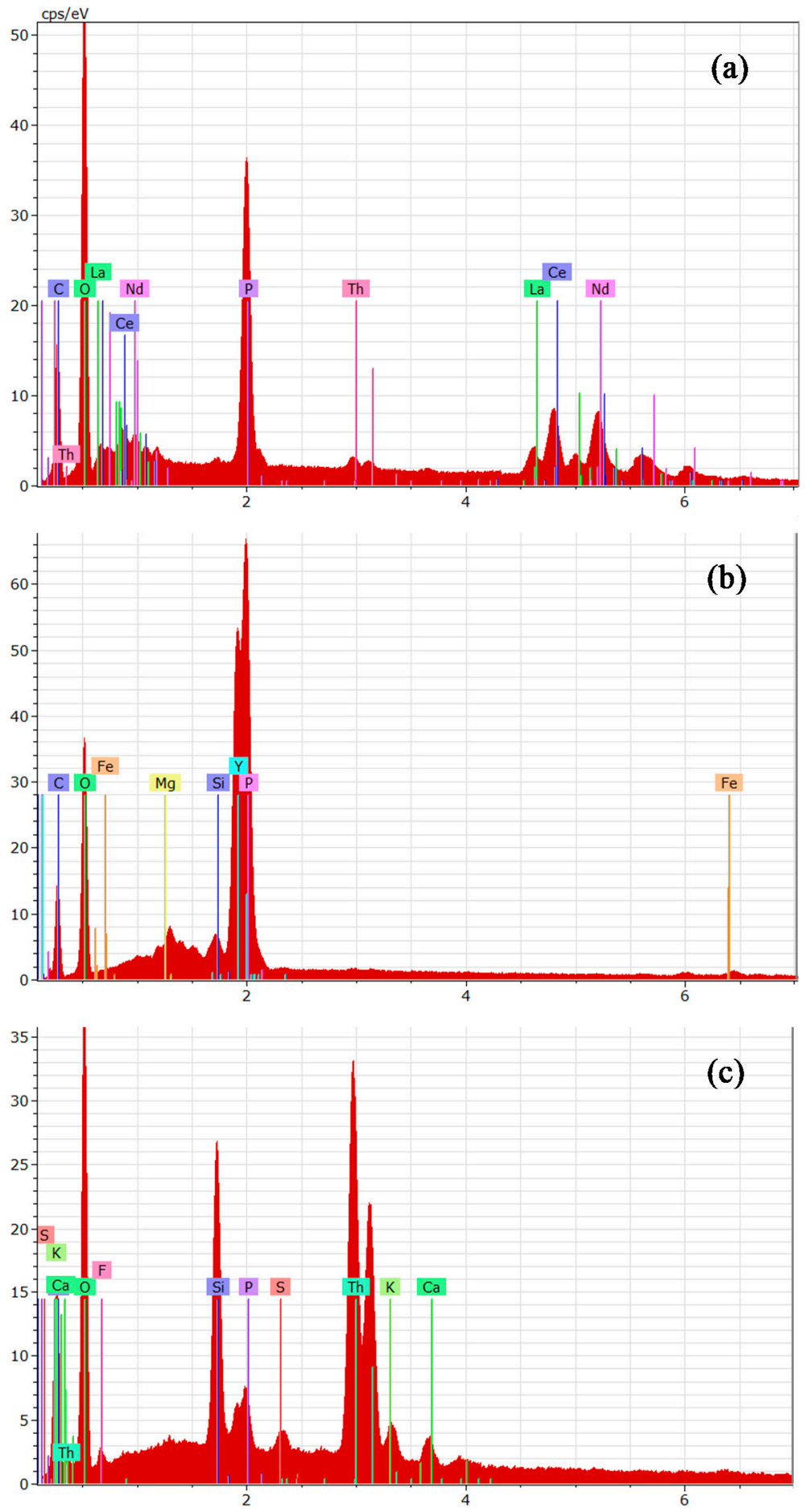

Figure 11. Examples of energy dispersive spectra (EDS) acquired from different parts of a crystal from sample 2a (see Figure 10): (a) monazite-(Ce), (b) xenotime-(Y), and (c) thorite, ideally $\mathrm{ThSiO}_{4}$.

\subsection{Radiation-Induced Transition from Monazite-(Ce) to Xenotime-(Y) in Sample $2 a$}

The chemical ages have been calculated using the concentrations of $\mathrm{Th}, \mathrm{U}$, and $\mathrm{Pb}$ (ppm). The age determination method was explained [23]. The internal radiation doses received have been calculated based on Equation (2) from Murakami et al. [24], which is modified from Holland and Gottfried [25]. Both samples received a significant amount of radiation doses of $4.68 \times 10^{16}$ and $1.93 \times 10^{17} \alpha$-decay events $/ \mathrm{mg}$, respectively (Table 4 ).

Radiation damaged signatures are found in small isolated domains in natural monazite [58,59]. However, our study indicates the redistribution of $A$ site cations in monazite. 
The driving thermal energy for the redistribution of cations comes from the internal radiations of ${ }^{238} \mathrm{U}$ and ${ }^{232} \mathrm{Th}$. The critical temperatures for the amorphization of monazite and zircon are about $430 \mathrm{~K}$ and $1100 \mathrm{~K}$, respectively [18]. Between $373-473 \mathrm{~K}$, natural monazite has the ability to heal fast $[15,17]$.

The individual collisions between the internal radiations and crystal structural framework are so complex that it is almost impossible to predict the exact mechanisms in natural geological settings. However, recent advancement of analytical techniques and computer simulation helps us to understand the mechanism of radiation-induced changes in a crystal. When a radioactive decay event occurs in a mineral, a significant amount of thermal energy can be produced. Various changes may occur when any mineral experiences internal radiations. The changes are mainly metastable and may depend on bond strength, the availability of structural voids, or spaces for the displaced atoms, intensity of radiation, and the chemical characteristics of the mineral [60]. After recrystallization, physical properties and crystallographic orientation can be reverted to its original state when healed. Sometimes the affected area may recrystallize a new phase with distinct crystal structural parameters that are different from the original [61].

The presence of three phases in sample $2 a$ is the result of internal radiation events. Since monazite has tremendous ability to recrystallize, no amorphous domains are retained. Depending on the available cations and amount of internal radiation doses during the recrystallization events, the volume of the affected area can recrystallize as the same phase and retains its original space group, but has distinct unit-cell parameters, bond distances, and angles, as observed for monazite phases $2 a$ and $2 b$, or a separate phase $2 c$ with higher symmetry and different structural parameters, as found for xenotime-( $(\mathrm{Y})$.

\section{Conclusions}

The HRPXRD data shows that a pegmatitic sample 2a contains three phases: two monazite-(Ce) phases and a xenotime-(Y) phase. Since the pegmatitic sample received a high amount of $\alpha$-radiation doses, a transition from monazite-(Ce) to xenotime- $(Y)$ may arise from the effects of radiation and redistribution of elements in the A site. However, other chemical processes cannot be ruled out as a cause for the transition. Although monazite-(Sm) contains a single phase, its structure is affected by strain, as indicated by the variable FWHM values of some HRPXRD peaks. The strain in the monazite-(Sm) crystal may arise from remnants of radiation damage.

Author Contributions: M.M.Z. carried out the EPMA experiment and S.M.A. carried out the HRPXRD experiment. Both authors analyzed the HRPXRD data and contributed to writing and editing the manuscript. All authors have read and agreed to the published version of the manuscript.

Funding: This research was funded by a NSERC Discovery Grant to SMA, grant number 10013896.

Acknowledgments: We thank the Academic Editor and three anonymous reviewers for useful comments that helped improve this paper. We thank Robert Marr for his help with EPMA data collection. The HRPXRD data were collected at the X-ray Operations and Research beamline 11-BM, Advanced Photon Source (APS), Argonne National Laboratory (ANL). Use of the APS was supported by the U.S. Dept. of Energy, Office of Science, Office of Basic Energy Sciences, under Contract No. DE-AC02-06CH11357.

Conflicts of Interest: The authors declare no conflict of interest.

\section{References}

1. Seydoux-Guillaume, A.M.; Wirth, R.; Heinrich, W.; Montel, J.M. Experimental determination of Thorium partitioning between monazite and xenotime using analytical electron microscopy and X-ray diffraction Rietveld analysis. Eur. J. Mineral. 2002, 14, 869-878. [CrossRef]

2. Pabst, A.; Hutton, C.O. Huttonite a new monoclinic thorium silicate. Am. Mineral. 1951, 36, 60-69.

3. Smitts, G. (U, Th)-bearing silicates in reefs of the Witwatersrand, South Africa. Can. Mineral. 1989, $27,643-655$.

4. Zaman, M.M.; Antao, S.M. Crystal chemistry and structural variations for zircon samples from various localities. Minerals 2020, 10, 947. [CrossRef] 
5. Zaman, M.M.; Antao, S.M. Crystal structure refinements of four monazite samples from different localities. Minerals 2020, 10 , 1028. [CrossRef]

6. Murata, K.J.; Rose, H.J., Jr.; Carron, M.K. Systematic variation of rare earths in monazite. Geochim. Cosmochim. Acta 1953, 4, 292-300. [CrossRef]

7. Spear, F.S.; Pyle, J.M. Apatite, monazite, and xenotime in metamorphic rocks. In Phosphates: Geochemical, Geobiological, and Materials Importance; Kohn, M.J., Rakovan, J., Hughes, J.M., Eds.; Mineralogical Society of America: Chantilly, VA, USA, 2002; Volume 48, pp. 293-335.

8. Fleischer, M.; Altschuler, Z.S. The relationship of the rare-earth composition of minerals to geological environment. Geochim. Cosmochim. Acta 1969, 33, 725-732. [CrossRef]

9. Rapp, R.P.; Watson, E.B. Monazite solubility and dissolution kinetics-Implications for the thorium and light rare-earth chemistry of felsic magmas. Contrib. Mineral. Petrol. 1986, 94, 304-316. [CrossRef]

10. Beall, G.W.; Boatner, L.A.; Mullica, D.F.; Milligan, W.O. The structure of cerium orthophosphate, a synthetic analog of monazite. J. Inorg. Nucl. Chem. 1981, 43, 101-105. [CrossRef]

11. Ni, Y.; Hughes, J.M.; Mariano, A.N. Crystal chemistry of the monazite and xenotime structures. Am. Mineral. 1995, 80, 21-26. [CrossRef]

12. Boatner, L.A. Synthesis, structure and properties of monazite, pretulite and xenotime. Rev. Mineral. Geochem. 2002, 48, 87-120. [CrossRef]

13. Ewing, R.C.; Meldrum, A.; Wang, L.; Weber, W.J.; Corrales, L.R. Radiation effects in zircon. Rev. Mineral. Geochem. 2003, 53, 387-425. [CrossRef]

14. Nasdala, L.; Kronz, A.; Hanchar, J.M.; Tichomirowa, M.; Davis, D.W.; Hofmeister, W. Effects of natural radiation damage on back-scattered electron images of single-crystals of minerals. Am. Mineral. 2006, 91, 1739-1746. [CrossRef]

15. Seydoux-Guillaume, A.M.; Wirth, R.; Nasdala, L.; Gottschalk, M.; Montel, J.M.; Heinrich, W. An XRD, TEM and Raman study of experimentally annealed natural monazite. Phys. Chem. Miner. 2002, 29, 240-253. [CrossRef]

16. Karkhanavala, M.D.; Shankar, J. An X-ray study of natural monazite: I. Proc. Indian Acad. Sci. 1954, A40, 67-71. [CrossRef]

17. Boatner, L.A. Monazite. In Radioactive Waste Forms for the Future; Lutze, W., Ewing, R.C., Eds.; Elsevier: Amsterdam, The Netherlands, 1988; pp. 495-564.

18. Meldrum, A.; Wang, L.M.; Ewing, R.C. Ion-beam-induced amorphization of monazite. Nucl. Instrum. Methods Phys. Res. 1996, B116, 220-224. [CrossRef]

19. Seydoux-Guillaume, A.M.; Wirth, R.; Deutsch, A.; Schärer, U. Microstructure of 24-1928 Ma concordant monazites; implications for geochronology and nuclear waste deposits. Geochim. Cosmochim. Acta 2004, 68, 2517-2527. [CrossRef]

20. Ewing, R.C.; Meldrum, A.; Wang, L.; Wang, S. Radiation-induced amorphization. In Transformation Processes in Minerals; Redfern, S.A.T., Carpenter, M.A., Eds.; Mineralogical Society of America: Washington, DC, USA, 2000; Volume 39, pp. 319-361.

21. Zaman, M.; Schubert, M.; Antao, S. Elevated radionuclide concentrations in heavy mineral-rich beach sands in the Cox's Bazar region, Bangladesh and related possible radiological effects. Isot. Environ. Health Stud. 2012, 48, 512-525. [CrossRef]

22. Pyle, J.M.; Spear, F.S.; Wark, D.A. Electron microprobe analysis of REE in apatite, monazite, and xenotime: Protocols and pitfalls. Rev. Mineral. Geochem. 2002, 48, 337-362. [CrossRef]

23. Montel, J.M.; Foret, S.; Veschambre, M.; Nicollet, C.; Provost, A. Electron microprobe dating of monazite. Chem. Geol. 1996, 131, 37-53. [CrossRef]

24. Murakami, T.; Chakoumakos, B.C.; Ewing, R.C.; Lumpkin, G.R.; Weber, W.J. Alpha-decay event damage in zircon. Am. Mineral. 1991, 76, 1510-1532.

25. Holland, H.D.; Gottfried, D. The effect of nuclear radiation on the structure of zircon. Acta Crystallogr. 1955, 8, 291-300. [CrossRef]

26. Antao, S.M.; Hassan, I.; Wang, J.; Lee, P.L.; Toby, B.H. State-of-the-art high-resolution powder X-ray diffraction (HRPXRD) illustrated with Rietveld structure refinement of quartz, sodalite, tremolite, and meionite. Can. Mineral. 2008, 46, 1501-1509. [CrossRef]

27. Lee, P.L.; Shu, D.; Ramanathan, M.; Preissner, C.; Wang, J.; Beno, M.A.; Von Dreele, R.B.; Ribaud, L.; Kurtz, C.; Antao, S.M.; et al. A twelve-analyzer detector system for high-resolution powder diffraction. J. Synchrotron Radiat. 2008, 15, 427-432. [CrossRef] [PubMed]

28. Wang, J.; Toby, B.H.; Lee, P.L.; Ribaud, L.; Antao, S.M.; Kurtz, C.; Ramanathan, M.; Von Dreele, R.B.; Beno, M.A. A dedicated powder diffraction beamline at the advanced photon source: Commissioning and early operational results. Rev. Sci. Instrum. 2008, 79, 085105. [CrossRef] [PubMed]

29. Antao, S.M.; Dhaliwal, I. Growth Oscillatory Zoning in Erythrite, Ideally $\mathrm{Co}_{3}\left(\mathrm{AsO}_{4}\right)_{2} \cdot 8 \mathrm{H}_{2} \mathrm{O}$ : Structural Variations in VivianiteGroup Minerals. Minerals 2017, 7, 136. [CrossRef]

30. Antao, S.M.; Hassan, I.; Crichton, W.A.; Parise, J.B. Effects of high pressure and temperature on cation ordering in magnesioferrite, $\mathrm{MgFe}_{2} \mathrm{O}_{4}$, using in situ synchrotron X-ray powder diffraction up to $1430 \mathrm{~K}$ and $6 \mathrm{GPa}$. Am. Mineral. 2005, 90, 1500-1505. [CrossRef]

31. Antao, S.M.; Hassan, I.; Mulder, W.H.; Lee, P.L. The $R-3 c \rightarrow R-3 m$ transition in nitratine, $\mathrm{NaNO}_{3}$, and implications for calcite, $\mathrm{CaCO}_{3}$. Phys. Chem. Miner. 2008, 35, 545-557. [CrossRef]

32. Ehm, L.; Michel, F.M.; Antao, S.M.; Martin, C.D.; Lee, P.L.; Shastri, S.D.; Chupas, P.J.; Parise, J.B. Structural changes in nanocrystalline mackinawaite (FeS) at high pressure. J. Appl. Crystallogr. 2009, 42, 15-21. [CrossRef] 
33. Hassan, I.; Antao, S.M.; Hersi, A.A. Single-crystal XRD, TEM, and thermal studies of the satellite reflections in nepheline. Can. Mineral. 2003, 41, 759-783. [CrossRef]

34. Hassan, I.; Antao, S.M.; Parise, J.B. Haüyne: Phase transition and high-temperature structures obtained from synchrotron radiation and Rietveld refinements. Mineral. Mag. 2004, 68, 499-513. [CrossRef]

35. Parise, J.B.; Antao, S.M.; Michel, F.M.; Martin, C.D.; Chupas, P.J.; Shastri, S.; Lee, P.L. Quantitative high-pressure pair distribution function analysis. J. Synchrotron Radiat. 2005, 12, 554-559. [CrossRef] [PubMed]

36. Rietveld, H.M. A profile refinement method for nuclear and magnetic structures. J. Appl. Crystallogr. 1969, 2, 65-71. [CrossRef]

37. Larson, A.C.; Von Dreele, R.B. General Structure Analysis System (GSAS), Los Alamos National Laboratory Report LAUR, 86-748; Los Alamos National Laboratory: Los Alamos, NM, USA, January 2000.

38. Toby, B.H. Expgui, a graphical user interface for GSAS. J. Appl. Crystallogr. 2001, 34, 210-213. [CrossRef]

39. Finger, L.W.; Cox, D.E.; Jephcoat, A.P. A correction for powder diffraction peak asymmetry due to axial divergence. J. Appl. Crystallogr. 1994, 27, 892-900. [CrossRef]

40. Clavier, N.; Podor, R.; Dacheux, N. Crystal chemistry of the monazite structure. J. Eur. Ceram. Soc. 2011, 31, 941-976. [CrossRef]

41. Hoshino, M.; Watanabe, Y.; Ishihara, S. Crystal chemistry of monazite from the granitic rocks of Japan: Petrographic implications. Can. Mineral. 2012, 50, 1331-1346. [CrossRef]

42. Van-Emden, B.; Graham, J.; Lincoln, F.J. The incorporation of actinides in monazite and xenotime from placer deposits in Western Australia. Can. Mineral. 1997, 35, 95-104.

43. Broska, I.; Petrík, I.; Williams, C.T. Coexisting monazite and allanite in peraluminous granitoids of the Tribeč Mountains, western Carpathians. Am. Mineral. 2000, 85, 22-32. [CrossRef]

44. Antao, S.M. Three cubic phases intergrown in a birefringent andradite-grossular garnet and their implications. Phys. Chem. Miner. 2013, 40, 705-716. [CrossRef]

45. Antao, S.M. The mystery of birefringent garnet: Is the symmetry lower than cubic? Powder Diffr. 2013, 28, 281-288. [CrossRef]

46. Antao, S.M.; Klincker, A.M. Origin of birefringence in andradite from Arizona, Madagascar, and Iran. Phys. Chem. Miner. 2013, 40, 575-586. [CrossRef]

47. Antao, S.M.; Mohib, S.; Zaman, M.; Marr, R.A. Ti-rich andradites: Chemistry, structure, multi-phases, optical anisotropy, and oscillatory zoning. Can. Mineral. 2015, 53, 133-158. [CrossRef]

48. Antao, S.M. Is near-endmember birefringent grossular non-cubic? New evidence from synchrotron diffraction. Can. Mineral. 2013, 51, 771-784. [CrossRef]

49. Antao, S.M. Crystal structure of morimotoite from Ice River, Canada. Powder Diffr. 2014, 29, 325-330. [CrossRef]

50. Antao, S.M.; Klincker, A.M. Crystal structure of a birefringent andradite-grossular from Crowsnest Pass, Alberta, Canada. Powder Diffr. 2014, 29, 20-27. [CrossRef]

51. Antao, S.M.; Round, S.A. Crystal chemistry of birefringent spessartine. Powder Diffr. 2014, 29, 233-240. [CrossRef]

52. Antao, S.M.; Hassan, I. A two-phase intergrowth of genthelvite from Mont Saint-Hilaire, Quebec. Can. Mineral. 2010, 48, 1217-1223. [CrossRef]

53. Antao, S.M. Crystal chemistry of birefringent hydrogrossular. Phys. Chem. Miner. 2015, 42, 455-474. [CrossRef]

54. Masau, M.; Černý, P.; Cooper, M.A.; Chapman, R.; Grice, J.D. Monazite-(Sm), a new member of the monazite group from the Annie claim \#3 granite pegmatite, southeastern Manitoba. Can. Mineral. 2002, 40, 1649-1655.

55. Delhez, R.; de Keijser, T.H.; Langford, J.I.; Louër, D.; Mittemeijer, E.J.; Sonneveld, E.J. The Rietveld Method; Young, R.A., Ed.; Oxford University Press: Oxford, UK, 1993; pp. 132-166.

56. Shannon, R.D. Revised effective ionic radii and systematic studies of interatomic distances in halides and chalcogenides. Acta Crystallogr. 1976, A32, 751-767. [CrossRef]

57. Ali, M.A. Mineral chemistry of monazite-(Nd), xenotime-(Y), apatite, fluorite and zircon hosting in lamprophyre dyke in Abu Rusheid area, South Eastern Desert, Egypt. Geologija 2012, 55, 93-105. [CrossRef]

58. Black, L.P.; Fitzgerald, J.D.; Harley, S.L. Pb isotopic composition, colour, and microstructure of monazites from a polymetamorphic rock in Antarctica. Contrib. Mineral. Petrol. 1984, 85, 141-148. [CrossRef]

59. Meldrum, A.; Boatner, L.A.; Weber, W.J.; Ewing, R.C. Radiation damage in zircon and monazite. Geochim. Cosmochim. Acta 1998, 62, 2509-2520. [CrossRef]

60. Kinchin, G.H.; Pease, R.S. The displacement atoms in solids by radiation. Rep. Prog. Phys. 1955, 18, 1-51. [CrossRef]

61. Pabst, A. The metamict state. Am. Mineral. 1952, 37, 137-157. 